\title{
A determining form for the two-dimensional Navier-Stokes equations: The Fourier modes case
}

\author{
Ciprian Foias, ${ }^{1, a)}$ Michael S. Jolly, ${ }^{2, b)}$ Rostyslav Kravchenko, ${ }^{3, c)}$ \\ and Edriss S. Titit ${ }^{4, d)}$ \\ ${ }^{1}$ Department of Mathematics, Texas A\&M University, College Station, Texas 77843, USA \\ ${ }^{2}$ Department of Mathematics, Indiana University, Bloomington, Indiana 47405, USA \\ ${ }^{3}$ Department of Mathematics, Chicago University, Chicago, Illinois 60637, USA \\ ${ }^{4}$ Department of Mathematics and Department of Mechanical and Aerospace Engineering, \\ University of California, Irvine, California 92697, USA and Department of Computer \\ Science and Applied Mathematics, Weizmann Institute of Science, Rehovot 76100, Israel
}

(Received 28 August 2012; accepted 23 October 2012; published online 27 November 2012)

\begin{abstract}
The determining modes for the two-dimensional incompressible Navier-Stokes equations (NSE) are shown to satisfy an ordinary differential equation (ODE) of the form $d v / d t=F(v)$, in the Banach space, $X$, of all bounded continuous functions of the variable $s \in \mathbb{R}$ with values in certain finite-dimensional linear space. This new evolution ODE, named determining form, induces an infinite-dimensional dynamical system in the space $X$ which is noteworthy for two reasons. One is that $F$ is globally Lipschitz from $X$ into itself. The other is that the long-term dynamics of the determining form contains that of the NSE; the traveling wave solutions of the determining form, i.e., those of the form $v(t, s)=v_{0}(t+s)$, correspond exactly to initial data $v_{0}$ that are projections of solutions of the global attractor of the NSE onto the determining modes. The determining form is also shown to be dissipative; an estimate for the radius of an absorbing ball is derived in terms of the number of determining modes and the Grashof number (a dimensionless physical parameter). (C) 2012 American Institute of Physics. [http://dx.doi.org/10.1063/1.4766459]
\end{abstract}

Dedicated to Professor Peter Constantin on the occasion of his 60th birthday

\section{INTRODUCTION}

The notion of determining modes ${ }^{5,7,18,19,25,27}$ was introduced as a way to gauge the number of degrees of freedom for the Navier-Stokes equations (NSE). Determining modes can be described as a (finite) subset of Fourier modes, such that for any solution $\{u(t): t \in \mathbb{R}\}$ in the global attractor $\mathcal{A}$ its projection into those modes, denoted by $\{v(t): t \in \mathbb{R}\}$, determines the complete solution. In particular, the projection of $\{u(t): t \in \mathbb{R}\}$ into the higher modes, denoted by $\{w(t): t \in \mathbb{R}\}$, is determined uniquely by $\{v(t): t \in \mathbb{R}\}$. Thus, there is a well-defined map $W: v(\cdot) \mapsto w(\cdot)$, which will play a major role in this paper. The number of determining modes is associated with the spatial complexity of the long-time dynamics, and hence suggests the resolution needed for numerical computations and data assimilations (see, e.g., Refs. 26 and 28). In this sense, estimates for number of determining modes is used much like those for the dimension of the global attractor. ${ }^{6,9,30}$

If an inertial manifold exists, the high wavenumber spatial modes are enslaved by a finite set of low wavenumber modes. ${ }^{6,8,20,21,30}$ As a consequence, if the low wavenumber modes are specified at just one point in time, the high wavenumber modes are determined for all time. In the case of an

\footnotetext{
a)E-mail: foias@math.tamu.edu.

b)E-mail: msjolly@indiana.edu.

c) Author to whom correspondence should be addressed. Electronic mail: rkchenko@gmail.com.

d) Fellow of the Center of Smart Interfaces (CSI), Technische Universität Darmstadt, Germany. Electronic mail: etiti@math.uci.edu.
} 
inertial manifold, the low modes satisfy an ordinary differential equation (ODE) called the inertial form whose phase space is finite-dimensional. The existence of an inertial manifold for the NSE, however, remains open.

Since a finite set of modes, if specified for all time, can determine the solutions to the NSE, a natural question is whether they satisfy an ordinary differential equation in a phase space of trajectories, the dynamics of which is consistent with those of the NSE. In other words, can one find an analog of the inertial form for determining modes? We show in Sec. IV that the determining modes $v$ for the NSE indeed satisfy a determining form, which is an ordinary differential equation of the form $d v / d t=F(v)$, where $F: X \rightarrow X$ is a globally Lipschitz map, and $X$ is the Banach space of all bounded continuous functions from $\mathbb{R}$ into the linear finite-dimensional space of the low wavenumber modes, given in (2.15). The key step is to extend beyond the global attractor the enslavement of the high modes mapping $W: v(\cdot) \mapsto w(\cdot)$, defined above, to the space $X$. This is done by analyzing a differential equation satisfied by $w(\cdot)$, and establishing the Lipschitz property of $W$. We then show in Sec. V that the resulting determining form, $d v / d t=F(v)$, is dissipative, and estimate the radius of its absorbing ball in terms of the number of determining modes, and the Grashof number (a dimensionless physical parameter given in (2.7)).

Though the number of determining modes is finite, the ODE, they satisfy, i.e., the determining form, induces an infinite-dimensional dynamical system, since it is an evolution ODE in the infinitedimensional Banach space $X$. Just as the set of partial differential equations that comprise the incompressible NSE can be written as an evolution equation, involving unbounded operators, in a Hilbert space $H$ of functions in the spatial variable, the determining modes evolve as solutions to an ODE in a Banach space $X$ of functions in an auxiliary time variable $s$. A significant difference, however, is that the vector field that governs the evolution in the determining form ODE is a globally Lipschitz map from the space $X$ into itself, whereas in the case of the NSE evolution equation it involves unbounded operators. Rather than being a dimension reduction of the NSE, the determining form is in fact an embedding of the original dynamics in a larger phase space. In the phase space $X$, the solutions at any moment in time, $t$, represent complete trajectories $v(t, s),-\infty<s<\infty$.

For certain initial trajectories, the behavior of solutions to the determining form is simple to describe. We show in Sec. VI that traveling wave solutions to the determining form can be characterized as those satisfying $v(t, s)=\mathfrak{p}\left(t+s ; u_{0}\right),-\infty<s<\infty$, where $\mathfrak{p}\left(s ; u_{0}\right)$ denotes the projection into the determining modes of the solution at time $s$ to the NSE with initial condition $u_{0} \in \mathcal{A}$. As shown in Sec. IV any solution with an initial trajectory $v(0, s),-\infty<s<\infty$ which is periodic in $s$, with period $T$, remains periodic in $s$, with period $T$, regardless of whether this initial trajectory is on the global attractor of the NSE. As a special case, any solution with an initial trajectory independent of $s$ remains so; if $v(0, s)=c(0),-\infty<s<\infty$, then $v(t, s)=c(t),-\infty<s<\infty$.

We also compare the general long time behavior of the determining form with that of the NSE. In Sec. VIII, we consider the sequences $v_{n}(t, s)=v\left(t+t_{n}, s-\left(t+t_{n}\right)\right)$ and $w_{n}(t, s)=w\left(t+t_{n}, s-\left(t+t_{n}\right)\right)$. We show that $v_{n}+w_{n}$ converges weakly to a solution of the NSE with an additional Reynolds force which has only finitely many modes. The stationary solutions of the determining form satisfy a differential algebraic system: an algebraic relation for $v$, coupled with a differential equation for $w$. Analogs of the energy and enstrophy balances are used in Sec. IX to show that, as in the case of the NSE, a certain extremal condition on norms of the stationary solution occur only for forces that are eigenfunctions of the Stokes operator.

Over the years it was proven that there are several other finite systems of parameters, different than the modes considered here, which also determine the long-time behavior of the solutions to the Navier-Stokes equations (e.g., determining nodes, finite volumes averages, finite elements, etc. ${ }^{3,4,17,22,23,27}$ ). It is worth stressing that the determining form studied here uses only determining modes, on which the partial differential operators in the NSE act in an obvious manner; this action is seemingly quite involved for other systems of determining parameters. One also faces a similar problem in representing inertial manifolds in terms of determining parameters other than the Fourier modes. This challenging general parametrization issue of the inertial manifolds has been solved in Ref. 24 for the determining nodes case and later in Ref. 4 for the general interpolant case, including the finite elements case, etc. In a forthcoming paper, ${ }^{14}$ we will introduce a general, unified construction of determining forms based on any finite system of determining parameters. This unified 
approach has also been used in Refs. 1 and 2 in the context of continuous data assimilations and finite-dimensional feedback control, respectively.

\section{STATEMENT OF MAIN RESULTS}

Our results are for the incompressible Navier-Stokes equations

$$
\begin{aligned}
& \frac{\partial u}{\partial t}-v \Delta u+(u \cdot \nabla) u+\nabla p=\Phi, \\
& \operatorname{div} u=0, \\
& \int_{\Omega} u d x=0, \quad \int_{\Omega} \Phi d x=0, \\
& u\left(t_{0}, x\right)=u_{0}(x)
\end{aligned}
$$

subject to periodic boundary conditions with basic domain $\Omega=[0, L]^{2}$. This system can be written more compactly as

$$
\begin{aligned}
& \frac{d}{d t} u(t)+v A u(t)+B(u(t), u(t))=f, \\
& u(t) \in H, t \geq 0, \text { and } u(0)=u_{0},
\end{aligned}
$$

where $H$ is the closure in $L^{2}(\Omega)^{2}$ of the set of all $\mathbb{R}^{2}$-valued trigonometric polynomials $\phi$ such that

$$
\nabla \cdot \phi=0 \quad \text { and } \quad \int_{\Omega} \phi(x) d x=0 .
$$

The bilinear operator $B$ and force $f$ are defined as

$$
B(u, v)=\mathcal{P}((u \cdot \nabla) v), \quad f=\mathcal{P} \Phi,
$$

where $\mathcal{P}$ is the Helmholtz-Leray orthogonal projector of $L^{2}(\Omega)^{2}$ onto $H$, and where $u, v$ are sufficiently smooth. When the input to $B$ is lengthy, and repeated, for convenience we will use the shorthand notation

$$
B(u)=B(u, u) .
$$

Unless specified otherwise, $f \in H$.

The operator $A=-\Delta$ is self-adjoint, and its eigenvalues are of the form

$$
\left(\frac{2 \pi}{L}\right)^{2} k \cdot k, \quad \text { where } k \in \mathbb{Z}^{2} \backslash\{0\} .
$$

We denote these eigenvalues by

$$
0<\lambda_{0}=(2 \pi / L)^{2} \leq \lambda_{1} \leq \lambda_{2} \leq \cdots
$$

arranged in increasing order and counted according to their multiplicities, and write $w_{0}, w_{1}, w_{2}, \ldots$, for the corresponding normalized eigenvectors (i.e., $\left|w_{j}\right|=1$ and $A w_{j}=\lambda_{j} w_{j}$ for $j=0,1,2, \ldots$ ).

We will also use the space $V:=D\left(A^{1 / 2}\right)$ with natural norm denoted

$$
\|u\|=\left|A^{1 / 2} u\right|=\left(\int_{\Omega} \sum_{j=1}^{2} \frac{\partial}{\partial x_{j}} u(x) \cdot \frac{\partial}{\partial x_{j}} u(x) d x\right)^{1 / 2}=\left(\sum_{j=0}^{\infty} \lambda_{j}\left(u, w_{j}\right)^{2}\right)^{2} .
$$

Since the boundary conditions are periodic, we can express any element in $H$ as a Fourier series

$$
u(x)=\sum_{k \in \mathbb{Z}^{2}} \hat{u}_{k} e^{i \kappa_{0} k \cdot x},
$$

where

$$
\kappa_{0}=\lambda_{0}^{1 / 2}=\frac{2 \pi}{L}, \quad \hat{u}_{0}=0, \quad \hat{u}_{k}^{*}=\hat{u}_{-k},
$$


and due to incompressibility, $k \cdot \hat{u}_{k}=0$. We define the orthogonal projectors $P_{N}, Q_{N}, P_{N}$ : $H \rightarrow \operatorname{span}\left\{w_{j} \mid \lambda_{j} \leq\left(\kappa_{0} N\right)^{2}\right\}$, by

$$
P_{N} u=\sum_{|k| \leq N} \hat{u}_{k} e^{i \kappa_{0} k \cdot x},
$$

where $u$ has the expansion in (2.5), and $Q_{N}=I-P_{N}$.

The notion of determining modes is defined for solutions on the global attractor $\mathcal{A}$, the minimal compact set in $V$ which attracts uniformly all solutions of the Navier-Stokes equations starting from the absorbing ball $\left\{h \in V:\|h\| \leq 2 G \nu \kappa_{0}\right\}$, where

$$
G=\frac{|f|}{v^{2} \kappa_{0}^{2}}
$$

is the generalized Grashof number introduced in Ref. 18. Recall that this set can also be characterized as

$$
\mathcal{A}=\left\{u_{0} \in H: \exists \quad u\left(t, u_{0}\right)=\text { solution } \forall t \in \mathbb{R}, \sup _{t}\|u(t)\|<\infty\right\}
$$

Definition 2.1: The projection $P_{N}$ is called determining if (and only if) for any two solutions $u_{1}(t), u_{2}(t)(t \in \mathbb{R})$ in $\mathcal{A}$ we have

$$
P_{N} u_{1}(t) \equiv P_{N} u_{2}(t) \Longrightarrow u_{1}(t) \equiv u_{2}(t)
$$

This is equivalent to the original definition in which the high mode trajectories are determined by the limit as $t \rightarrow \infty$ of their projections. ${ }^{16,17,19}$ Using the original definition, it is shown in Ref. 27 that for $P_{N}$ to be determining, it is sufficient to take $N=O(G)$. For completeness, we reproduce that estimate using Definition 2.1 in the Appendix.

A determining projection defines a natural mapping. By denoting

$$
\mathfrak{q}(s)=W\left(s ; P_{N} u(\cdot)\right)=Q_{N} u(s) \quad(s \in \mathbb{R}),
$$

we have that on the set

$$
\left\{P_{N} u(\cdot): u(\cdot) \text { is a solution of }(2.1) \text { in } \mathcal{A}\right\}
$$

the map

$$
P_{N} u(\cdot) \mapsto \mathfrak{q}(s)=W\left(s ; P_{N} u(\cdot)\right) \quad(s \in \mathbb{R})
$$

is well-defined by the following properties:

$$
\begin{gathered}
\frac{d \mathfrak{q}(s)}{d s}+v A \mathfrak{q}(s)=Q_{N} f-Q_{N} B\left(P_{N} u(s)+\mathfrak{q}(s)\right), \quad \forall s \in \mathbb{R}, \\
\sup _{s \in \mathbb{R}}\|\mathfrak{q}(s)\|<\infty,
\end{gathered}
$$

where we used the shorthand notation in (2.3).

Now consider the Banach spaces

$$
\begin{aligned}
& X=C_{b}\left(\mathbb{R}, P_{N} H\right)=\left\{v: \mathbb{R} \mapsto P_{N} H, v(s) \text { continuous } \forall s \in \mathbb{R},\|v\|_{X}<\infty\right\}, \\
& Y=C_{b}\left(\mathbb{R}, Q_{N} H\right)=\left\{w: \mathbb{R} \mapsto Q_{N} H, w(s) \text { continuous } \forall s \in \mathbb{R},|w|_{Y}<\infty\right\},
\end{aligned}
$$

where

$$
\|v\|_{X}=\sup _{s}\|v(s)\|, \quad|w|_{Y}=\sup _{s}|w(s)|
$$


Let $\varphi(\xi)$ be a continuous, piecewise linear function, that is equal to 1 for $|\xi| \leq 2 G, 0$ for $|\xi| \geq 3 G$, where $G$ is the Grashof number given in (2.7), and consider

$$
\frac{d}{d t} v(t)+v A v(t)+P_{N} B(\varphi v(t)+W(v(t)))=P_{N} f,
$$

where

$$
\varphi=\varphi\left(\frac{\|v(t)\|}{\nu \kappa_{0}}\right)
$$

The first result introduces the main notion in this paper.

Theorem 2.2: For $N=O(G)$ (up to a logarithm, see (4.36)) the map in (2.12) can be extended from the set in (2.11) to a Lipschitz map $X \rightarrow Y$ so that (2.17) can be written as

$$
\frac{d v}{d t}=F(v) \quad(\forall t \in \mathbb{R}, v \in X)
$$

where $F: X \rightarrow X$ is globally Lipschitz, i.e.,

$$
\left\|F\left(v_{1}\right)-F\left(v_{2}\right)\right\|_{X} \leq L_{F}\left\|v_{1}-v_{2}\right\|_{X},
$$

and the constant $L_{F}$ is independent of $v_{1}, v_{2}$.

Thus, the Navier-Stokes equations on the attractor $\mathcal{A}$ is transformed into an ordinary differential equation in the space of trajectories $X$ defined in (2.15). We refer to this ODE as the determining form. Like the NSE itself, the determining form is dissipative.

Theorem 2.3: For $N=O(G)$ (up to a logarithm) there is a ball centered at the origin, with radius $O\left(G^{2}\right)$, which is an absorbing ball in $X$ for (2.17).

While the above estimate for the radius of the absorbing ball for the determining is much larger than that for the NSE, the two can be brought closer if $N$ is taken larger (see Remark 5.1).

The long-time behavior of the determining form remains open. Let $\mathcal{B}_{r}$ be an absorbing ball in $X$, centered at 0 with radius $r>r_{0}$, and let

$$
\mathcal{M}=\cap_{t>0} S_{\mathrm{DF}}(t) \mathcal{B}_{r}
$$

where $S_{\mathrm{DF}}$ denotes the solution operator for (2.17), i.e., $S_{\mathrm{DF}}(t) v_{0}=v(t)$, where $v(t)$ is the solution with initial value $v(0)=v_{0}$. Note that $\mathcal{M}$ is independent of $r: v_{0} \in X$ belongs to $\mathcal{M}$ if and only if there is a bounded solution $v:(-\infty, \infty) \rightarrow X$ of (4.5) defined on the whole real line such that $v(0)=v_{0}$. We can also characterize $\mathcal{M}$ as the maximal bounded set that is invariant (i.e., $S_{\mathrm{DF}}(t) \mathcal{M}=\mathcal{M} \forall t \geq 0$ ), both forward and backward in time, under the flow generated by (2.17). We do not know if $\mathcal{M}$ is compact in $X$, though we suspect it is compact in an appropriate, larger space. We do know that $\mathcal{M}$ contains the global attractor of the NSE since solutions in $\mathcal{A}$ can be characterized as traveling wave solutions for the determining form (2.17).

Theorem 2.4: For $N=O(G)$ (up to a logarithm) a solution $v(t)$ of (2.17) is a traveling wave solution if and only if $v(0)$ is a projection of a solution of $(2.1)$ in $\mathcal{A}$.

For $v(t) \in X$ we denote by $v(t, s)$ the value of $v(t)$ at the parameter $s \in \mathbb{R}$.

Remark 2.1: Note that a function $v(t, s-t)$ being independent of $t$ is equivalent to $v(t, s)$ $=v(0, s+t)$, for all $s, t$; thus if $\|v(0)\|_{X} \leq 2 v \kappa_{0} G$, then $v(t)$ is the $P_{N}$-projection of a solution on the global attractor of the Navier-Stokes equations. 
Another link to the dynamics of the NSE is that periodicity of trajectories is conserved by (2.17).

Proposition 2.5: If $v(t)$ is the solution of (2.17) and $v(0)$ is a periodic trajectory with period $T$, then $v(t)$ is periodic with period $T$ for any $t$. In particular, if $v(0)$ is constant trajectory, then $v(t)$ is constant for any $t$.

Recall that $u_{0} \in H$ is stationary for (2.1) if

$$
v A u_{0}+B\left(u_{0}, u_{0}\right)=f .
$$

Clearly, if

$$
v(0, \cdot)=P_{N} u_{0}(\cdot),
$$

then $v(t, \cdot)=P_{N} u_{0}(\cdot)$ for all $t$ is a stationary solution of (2.17). In fact by Theorem 2.4 any stationary solution $v(t, s)$ of (2.17) which is constant in $s$ must satisfy (2.22). The set of all stationary solutions of (2.17) may, however, be much larger than the set of constant functions in $X$ that are the projections of the stationary solutions of (2.1) as in (2.22). In fact, we show in Ref. 13 that a determining form for the Lorenz system has stationary solutions which are not in the global attractor of the Lorenz system.

\section{SPECIFIC PRELIMINARIES}

We will use the following elementary estimates in several places.

Lemma 3.1: Let $x(t), y(t)$ be non-negative functions defined on the interval $I$, where $I=\left[t_{0}, t_{1}\right)$, for $t_{0}$ finite, or $\left(-\infty, t_{1}\right)$. Suppose that $x^{2}(t)$ is differentiable on $\left(t_{0}, t_{1}\right)$, and satisfies

$$
\frac{d}{d t} x^{2}(t) \leq-a y^{2}(t)+b y(t), \quad x(t) \leq \varepsilon y(t)
$$

for $t \in I$ where $a, b, \varepsilon$ are positive constants. Then the following hold:

(i) If $x(t) \geq \varepsilon$ bla for all $t \in\left[t_{0}, t_{1}\right)$, then

$$
x(t)-\varepsilon \frac{b}{a} \leq e^{-a\left(t-t_{0}\right) / 2 \varepsilon^{2}}\left(x\left(t_{0}\right)-\varepsilon \frac{b}{a}\right) ;
$$

(ii) if $x(\tilde{t}) \leq \varepsilon b / a$, for some $\tilde{t} \in I$, then $x(t) \leq \varepsilon b / a$ for all $t \in\left[\tilde{t}, t_{1}\right)$;

(iii) if $x(t)$ is bounded on $\left(-\infty, t_{1}\right)$, then $x(t) \leq \varepsilon$ b/a for all $t \in\left(-\infty, t_{1}\right)$.

Proof: To prove (i) define $c(t):=\varepsilon^{-1} x(t)-b / a$. Since

$$
y(t) \geq \frac{x(t)}{\varepsilon}=c(t)+\frac{b}{a} \geq \frac{b}{a},
$$

we have

$$
\begin{aligned}
& \frac{d c}{d t} \frac{2 \varepsilon^{2}}{a}(a c+b)=2 \varepsilon^{2} c \frac{d c}{d t}+2 \varepsilon^{2} \frac{b}{a} \frac{d c}{d t}=\frac{d}{d t} x^{2} \leq-a y^{2}+b y \\
& \leq-a\left(c+\frac{b}{a}\right)^{2}+b\left(c+\frac{b}{a}\right)=-c(a c+b) .
\end{aligned}
$$

Thus, since $a c+b=a x / \varepsilon \geq b>0$,

$$
e^{a t / 2 \varepsilon^{2}} c(t) \leq e^{a t_{0} / 2 \varepsilon^{2}} c\left(t_{0}\right)
$$

and (i) follows.

To prove (ii), suppose that $x\left(t^{\prime}\right)>\varepsilon b / a$ for some $t^{\prime}>\tilde{t}$. It follows that both $\frac{d}{d t} x^{2}>0$ and $x>\varepsilon b / a$ hold at some time between $\tilde{t}$ and $t^{\prime}$. We have at that time by (3.3),

$$
0<\frac{d}{d t} x^{2} \leq-a y^{2}+b y \leq 0,
$$

a contradiction.

(iii) follows from (i). 
We also use the following immediate corollary of Lemma 3.1 for the case in which the coefficient $b$ varies with time.

Corollary 3.2: Suppose $x(t), y(t)$ are positive functions defined on the interval I, where I $=\left[t_{0}, t_{1}\right)$ for $t_{0}$ finite, or $\left(-\infty, t_{1}\right)$, and $\varepsilon>0$ is such that $x(t) \leq \varepsilon y(t)$ and

$$
\frac{d}{d t} x^{2}(t) \leq-a y^{2}(t)+b(t) y(t)
$$

for $t \in I$, with $a$, $b(t)$ positive, then

(i) if $x(\tilde{t}) \leq \varepsilon \sup _{t^{\prime} \leq \tilde{t}} b\left(t^{\prime}\right) / a$, for some $\tilde{t} \in I$, then $x(t) \leq \varepsilon \sup _{t^{\prime} \leq t} b\left(t^{\prime}\right) / a$ for all $t \in\left[\tilde{t}, t_{1}\right)$;

(ii) if $x(t)$ is bounded on $\left(-\infty, t_{1}\right)$, then $x(t) \leq \varepsilon$ bla for all $t \in\left(-\infty, t_{1}\right)$.

\section{THE DETERMINING FORM}

\section{A. Extending the domain of $W$ to $C_{b}\left(\mathbb{R}, P_{N} V\right)$}

To ultimately write a differential equation for the low mode trajectories, we need to extend the domain of $W$ beyond the projection of the global attractor into $P_{N} V$. We define

$$
\mathfrak{p}(t, s)=P_{N} u(s+t), \quad(\forall s, t \in \mathbb{R})
$$

and apply the projection $P_{N}$ to (2.1) to obtain

$$
\frac{\partial}{\partial t} \mathfrak{p}(t, s)=-v A \mathfrak{p}(t, s)+P_{N} f-P_{N} B\left(\mathfrak{p}(t, s)+W\left(t+s ; P_{N} u(\cdot)\right)\right) .
$$

But it is clear that

$$
W\left(t+s ; P_{N} u(\cdot)\right) \equiv W\left(s ; \tau_{t} P_{N} u(\cdot)\right) \equiv W(s ; \mathfrak{p}(t, \cdot)),
$$

where $\tau_{t}$ is the shift by $t$,

$$
\tau_{t} u(s)=u(s+t), \quad \text { for all } s \in \mathbb{R} .
$$

Thus (4.2) becomes

$$
\frac{\partial}{\partial t} \mathfrak{p}(t, s)=-v A \mathfrak{p}(t, s)+P_{N} f-P_{N} B(\mathfrak{p}(t, s)+W(s ; \mathfrak{p}(t, \cdot))) .
$$

Let $\mathfrak{p}(t, s)$ in (4.1) be written as $v(t) \in X$, so that (4.5) can be viewed as the "differential equation"

$$
\frac{d}{d t} v(t)=-v A v(t)+P_{N} f-P_{N} B(v(t)+W(v(t))) \quad(\forall t \in \mathbb{R}),
$$

where throughout $v(t)$ denotes the function $s \mapsto v(t, s)$ in $X$ and $W(v(t))$ denotes the function $s \mapsto W(s ; v(t))$. We should stress that $d v / d t$ in (4.6) is the derivative of a trajectory in the space $X$. It is also useful to note that, in particular, the following partial differential equation holds

$$
\frac{\partial}{\partial t} v(t, s)=P_{N} f-v A v(t, s)-P_{N} B(v(t, s)+W(s ; v(t, \cdot))),
$$

for every $s \in \mathbb{R}$, where $\partial / \partial t$ is taken in $H$. Note that (4.6) is defined only for those $v(t)$ that come from $\mathfrak{p}(t, s)$, i.e., from $u \in \mathcal{A}$.

Let $h \in Q_{N} H, v$ be an element of $X$. We will show that

$$
\frac{d}{d s} w+v A w+Q_{N} B(\varphi v+w, \varphi v+w)=h
$$

has a unique bounded solution $w(s) \in Y$. Consider a Galerkin approximation for $n>N$, of Eq. (4.7), namely,

$$
\begin{aligned}
& \frac{d}{d s} w_{n}+v A w_{n}+P_{n} Q_{N} B\left(\varphi v+w_{n}, \varphi v+w_{n}\right)=P_{n} h \\
& w_{n}(s) \equiv P_{n} w_{n}(s) \text { for } s \geq s_{0}
\end{aligned}
$$


with the initial condition $w_{n}\left(s_{0}\right)=0$. Since (4.8) is an ordinary differential equation, it has a unique solution $w_{n}(s)$ for $s$ in some maximal interval $\left[s_{0}, s_{1}\right)$, on which $\|w(s)\|<\infty$.

Taking the scalar product of (4.8) with $A w_{n}$ and using relation (A5), we obtain

$$
\frac{1}{2} \frac{d}{d s}\left\|w_{n}\right\|^{2}+v\left|A w_{n}\right|^{2}+\varphi^{2}\left(B(v, v), A w_{n}\right)-\varphi\left(B\left(w_{n}, w_{n}\right), A v\right)=\left(h, A w_{n}\right) .
$$

Note that it follows from the definition of $\varphi$ that

$$
\left[\varphi\left(\frac{\|v\|}{v \kappa_{0}}\right)\right]^{\alpha}\|v\| \leq 3 v \kappa_{0} G \quad \forall v \in X, \forall \alpha>0 .
$$

Using (A20) and (4.10), we have

$$
\varphi^{2}\left|\left(B(v, v), A w_{n}\right)\right| \leq \varphi^{2} c_{T}\|v\|^{2}\left|A w_{n}\right|(\ln e N)^{1 / 2} \leq 9 c_{T} v^{2} \kappa_{0}^{2} G^{2}(\ln e N)^{1 / 2}\left|A w_{n}\right|,
$$

while using (A21), $\left\|w_{n}\right\| \leq\left(1 / \kappa_{0} N\right)\left|A w_{n}\right|,\left|A^{3 / 2} v\right| \leq\left(\kappa_{0} N\right)^{2}\|v\|$, and (4.10), we get

$$
\begin{aligned}
& \left.\varphi\left|\left(B\left(w_{n}, w_{n}\right), A v\right)\right|=\varphi \mid\left(B\left(w_{n}, A v\right), w_{n}\right)\right)\left|\leq c_{\mathrm{L}}\right| w_{n}||\left|w_{n} \|\right| A^{3 / 2} v \mid \leq \\
& 3 c_{\mathrm{L}} v \frac{G}{N}\left|A w_{n}\right|^{2} .
\end{aligned}
$$

Thus

$$
\begin{aligned}
& \frac{1}{2} \frac{d}{d s}\left\|w_{n}\right\|^{2}+v\left|A w_{n}\right|^{2} \\
& \quad \leq|h|\left|A w_{n}\right|+\varphi^{2}\left|\left(B(v, v), A w_{n}\right)\right|+\varphi\left|\left(B\left(w_{n}, w_{n}\right), A v\right)\right| \\
& \quad \leq 3 c_{\mathrm{L}} v \frac{G}{N}\left|A w_{n}\right|^{2}+\left[\frac{|h|}{|f|} v^{2} \kappa_{0}^{2} G+9 c_{T} v^{2} \kappa_{0}^{2} G^{2}(\ln e N)^{1 / 2}\right]\left|A w_{n}\right| .
\end{aligned}
$$

Now we have by (ii) of Lemma 3.1 (with $\varepsilon=1 /\left(\kappa_{0} N\right)$ ), that if $N$ and $G$ are such that

$$
N>3 c_{\mathrm{L}} G
$$

then

$$
\|w(s)\| \leq v \kappa_{0} \frac{9 c_{T} G^{2}(\ln e N)^{1 / 2}+\frac{|h|}{|f|} G}{N-3 c_{\mathrm{L}} G} .
$$

In the following we assume that (4.12) is satisfied, and introduce the notation

$$
\beta(G, N)=\frac{9 c_{T} G(\ln e N)^{1 / 2}+\frac{|h|}{|f|}}{N-3 c_{\mathrm{L}} G} .
$$

Thus for the solution $w_{n}(s)$ of (4.8) with initial condition $w_{n}\left(s_{0}\right)=0$,

$$
\left\|w_{n}(s)\right\| \leq v \kappa_{0} G \beta(G, N) .
$$

It follows from the general theory of ordinary differential equations that $w_{n}$ can be extended to the solution of (4.8) defined on $\left[s_{0}, \infty\right)$. Therefore, the classical proofs of existence for solutions of Eq. (2.1) (see, e.g., Chap. 9 and Lemmas 8.2 and 8.4 in Chap. 8 of Ref. 6) apply almost verbatim to (4.7). It follows that (4.7) has a unique solution on $\left[s_{0}, \infty\right)$ satisfying $w\left(s_{0}\right)=0$. Now denoting by $w^{(n)}(\cdot)$ the solution of $(4.7)$ on $[-n, \infty)$ satisfying $w^{(n)}(-n)=0$, we have

$$
\left\|w^{(n)}(s)\right\| \leq \kappa_{0} v G \beta(G, N) \text { for all } s \in[-n, \infty)
$$


It is also easy to show using (4.11) that for any fixed, bounded interval $\left[s_{0}, s_{1}\right]$ we have

$$
\sup _{n} \int_{s_{0}}^{s_{1}}\left|A w^{(n)}(s)\right|^{2} d s<\infty .
$$

It is well known how to prove that there exists a subsequence $\left\{n_{j}\right\}_{j=1}^{\infty}$ such that $w^{\left(n_{j}\right)}$ converges to a solution $w(s)$ of (4.7) in $L^{2}\left(\left[s_{0}, s_{1}\right] ; V\right)$ satisfying

$$
\|w(s)\| \leq \kappa_{0} \nu G \beta(G, N) \text { for all } s \in\left[s_{0}, s_{1}\right],
$$

and moreover, there exists $C=C(G)$, such that for $s_{1}-s_{0}<1$,

$$
\int_{s_{1}}^{s_{2}}|A w(s)|^{2} \leq C \nu \kappa_{0}^{2} \nu G \text { for all } s_{1}, s_{2} \text { such that } s_{1}-s_{0}<1
$$

(see again Ref. 6).

We note that if $w(s)$ is any bounded solution of (4.7), defined on $\left(-\infty, s_{1}\right]$, then in exactly the same way we get the analog of (4.11) for $w$, from which by (iii) of Lemma 3.1 we get the bound

$$
\|w(s)\| \leq \nu \kappa_{0} G \beta(G, N) \quad \text { for all } s \in\left(-\infty, s_{1}\right] .
$$

We now prove that this solution, which is bounded in the $\|\cdot\|$ norm, is unique for sufficiently large $N$. Let $w_{1}, w_{2}$ be two bounded solutions of (4.7). Denote $w=w_{1}-w_{2}$. Then

$$
\frac{d}{d s} w+v A w+Q_{N} B\left(\varphi v+w_{1}, \varphi v+w_{1}\right)-Q_{N} B\left(\varphi v+w_{2}, \varphi v+w_{2}\right)=0
$$

Taking the scalar product of the above equation with $w$ we get

$$
\begin{aligned}
\frac{1}{2} \frac{d}{d s}|w|^{2} & +v\|w\|^{2} \\
& =-\left(B\left(\varphi v+w_{1}, \varphi v+w_{1}\right), w\right)+\left(B\left(\varphi v+w_{2}, \varphi v+w_{2}\right), w\right) \\
& =-\left(B\left(w, \varphi v+w_{1}\right), w\right)-\left(B\left(\varphi v+w_{2}, w\right), w\right)=-\left(B\left(w, \varphi v+w_{1}\right), w\right) .
\end{aligned}
$$

We apply (A21), (4.10), and (4.18) to obtain

$$
\left|\left(B\left(w, \varphi v+w_{1}\right), w\right)\right| \leq c_{\mathrm{L}}|w|\|w\|\left(\varphi\|v\|+\left\|w_{1}\right\|\right) \leq G(3+\beta(G, N)) c_{\mathrm{L}} \kappa_{0} v|w|\|w\|
$$

so that

$$
\frac{1}{2} \frac{d}{d s}|w|^{2}+v\|w\|^{2} \leq G(3+\beta(G, N)) c_{\mathrm{L}} \kappa_{0} v\|w\||w|
$$

Now we use

$$
|w| \leq \frac{1}{\kappa_{0} N}\|w\|
$$

to get

$$
\frac{d}{d s}|w|^{2}+2 \kappa_{0}^{2} v N^{2}\left(1-c_{\mathrm{L}} G \frac{3+\beta(G, N)}{N}\right)|w|^{2} \leq 0 .
$$

Since $|w|$ is bounded on $(-\infty, \infty)$, we find that $w=0$, when

$$
N>c_{\mathrm{L}} G(3+\beta(G, N)) .
$$

We next show that $w(s)$ is continuous in $s$. Integrating (4.7), we have

$$
\left|w\left(s_{2}\right)-w\left(s_{1}\right)\right|=\left|\int_{s_{1}}^{s_{2}} h-Q_{N} B(\varphi v(s)+w(s))-v A w(s) d s\right| .
$$


Using (A19), (A20), and (A23), together with (4.10) and (4.18), we find that

$$
\begin{gathered}
|B(\varphi v)| \leq 9 c_{\mathrm{A}}\left(\nu \kappa_{0}\right)^{2} G^{2}, \quad|B(\varphi v, w)| \leq 3 c_{B}\left(\nu \kappa_{0}\right)^{2} G^{2} \beta(G, N), \\
|B(w, \varphi v)| \leq 3 c_{T}\left(\nu \kappa_{0}\right)^{2} G^{2} \beta(G, N), \quad|B(w)| \leq \frac{c_{\mathrm{A}} \kappa_{0}}{N^{1 / 2}}(\nu G \beta(G, N))^{3 / 2}|A w|^{1 / 2} .
\end{gathered}
$$

It follows that (for $0<s_{2}-s_{1}<1$ ), we have

$$
\begin{aligned}
\left|w\left(s_{2}\right)-w\left(s_{1}\right)\right| \leq & \left\{|h|+\left[\left(9 c_{\mathrm{A}}+3 \beta(G, N)\left(c_{B}+c_{T}\right) G^{2}+C(G)\right]\left(\nu \kappa_{0}\right)^{2}\right\}\left(s_{2}-s_{1}\right)\right. \\
& +\frac{c_{\mathrm{A}}}{N^{1 / 2}} \kappa_{0}^{3 / 2} v^{7 / 4}(G \beta(G, N))^{3 / 2}[C(G)]^{1 / 4}\left(s_{2}-s_{1}\right)^{3 / 4}
\end{aligned}
$$

We work with both the $|\cdot|$ - and $\|\cdot\|$-norms to allow for $h$ (hence $f$ ) to be in $H$, rather than in $V$.

Note that (4.24) implies (4.12). This concludes the proof of the following lemma.

Lemma 4.1: Assume that $N$ satisfies (4.24), where $\beta(G, N)$ is defined in (4.14). Then Eq. (4.7) has a unique weak solution defined on the whole real line, which is bounded in the $\|\cdot\|$ norm. Moreover, this weak solution is in $Y$ with

$$
\|w(s)\| \leq \nu \kappa_{0} G \beta(G, N) \quad \forall s \in \mathbb{R} \quad \text { and } \quad|w|_{Y} \leq \nu G \frac{\beta(G, N)}{N} .
$$

This lemma allows us to define the (nonlinear) operator $W$ which takes $v \in X$ to the unique solution of the corresponding Eq. (4.7). By Lemma 4.1, we have that

$$
W\left(s ; \tau_{t}(v(\cdot))\right)=\tau_{t} W(s ; v(\cdot)) .
$$

It is also useful to have a bound for $|A w|$ if $\|h\|$ is finite. Consider the following a priori estimates of $A w$. Taking the scalar product of (4.7) with $A^{2} w$, we have

$$
\frac{1}{2} \frac{d}{d s}|A w|^{2}+v\left|A^{3 / 2} w\right|^{2}+\left(\left(B(\varphi v+w), A^{2} w\right)=\left(h, A^{2} w\right) \leq\|h\|\left|A^{3 / 2} w\right| .\right.
$$

Using (A6), expanding, then applying (A2) (three times) and (A4), we obtain

$$
\begin{aligned}
((B & \left.(\varphi v+w, \varphi v+w), A^{2} w\right) \\
& =\varphi^{2}(B(v, A v), A w)+\varphi(B(v, A w), A w)+\varphi(B(w, A v), A w)+(B(w, A w), A w) \\
& -\varphi^{2}(B(A v, v), A w)-\varphi(B(A v, w), A w)-\varphi(B(A w, v), A w)-(B(A w, w), A w) \\
& =-\varphi^{2}(B(v, A w), A v)-\varphi(B(w, A w), A v) \\
& -\varphi^{2}(B(A w, v), A v)-\varphi(B(A v, w), A w)-\varphi(B(A w, v), A w)-(B(A w, w), A w),
\end{aligned}
$$


so that by applying for the first term (A18) and for the rest of terms (A21), followed by (4.10) and (4.25), we have

$$
\begin{aligned}
\mid((B & \left.(\varphi v+w, \varphi v+w), A^{2} w\right) \mid \\
\leq & c_{T} \varphi^{2}\|v\|\left|A ^ { 3 / 2 } w \| A v | ( \operatorname { l n } e N ) ^ { 1 / 2 } + c _ { \mathrm { L } } \varphi | w | ^ { 1 / 2 } \| w \left\|^{1 / 2}\left|A^{3 / 2} w \| A v\right|^{1 / 2}\left|A^{3 / 2} v\right|^{1 / 2}\right.\right. \\
& +c_{\mathrm{L}} \varphi^{2}|A w|^{1 / 2}\left|A^{3 / 2} w\right|^{1 / 2}\|v\||A v|^{1 / 2}\left|A^{3 / 2} v\right|^{1 / 2} \\
& +c_{\mathrm{L}} \varphi|A v|^{1 / 2}\left|A^{3 / 2} v\right|^{1 / 2}\|w\||A w|^{1 / 2}\left|A^{3 / 2} w\right|^{1 / 2} \\
& +c_{\mathrm{L}} \varphi|A w|\left|A^{3 / 2} w\right|\|v\|+c_{\mathrm{L}}\left|A^{3 / 2} w\right||A w|\|w\| \\
\leq & c_{T}(\varphi\|v\|)^{2} \kappa_{0} N(\ln e N)^{1 / 2}\left|A^{3 / 2} w\right|+c_{\mathrm{L}} \varphi\|v\| \frac{\left|A^{3 / 2} w\right|^{2}}{\kappa_{0} N} \\
& +c_{\mathrm{L}}(\varphi\|v\|)^{2} \kappa_{0} N\left|A^{3 / 2} w\right|+c_{\mathrm{L}} \varphi\|v\| \frac{\left|A^{3 / 2} w\right|^{2}}{\kappa_{0} N} \\
& +c_{\mathrm{L}} \varphi\|v\| \frac{\left|A^{3 / 2} w\right|^{2}}{\kappa_{0} N}+c_{\mathrm{L}} \kappa_{0} v \beta(G, N) G \frac{\left|A^{3 / 2} w\right|^{2}}{\kappa_{0} N} \\
\leq & c_{\mathrm{L}}[9+\beta(G, N)] v \frac{G}{N}\left|A^{3 / 2} w\right|^{2}+9 v^{2} \kappa_{0}^{3} G^{2} N\left(c_{T}(\ln e N)^{1 / 2}+c_{\mathrm{L}}\right)\left|A^{3 / 2} w\right| .
\end{aligned}
$$

Thus if

$$
N>c_{\mathrm{L}} G(9+\beta(G, N)),
$$

we can apply Lemma 3.1 (iii) to obtain

$$
|A w| \leq \frac{\|h\|+9 v^{2} \kappa_{0}^{3} G^{2} N\left(c_{T}(\ln e N)^{1 / 2}+c_{\mathrm{L}}\right)}{\nu \kappa_{0}\left\{N-c_{\mathrm{L}} G(9+\beta(G, N))\right\}} .
$$

Using the Galerkin approximation we get the bound (4.29) for $w_{n}$, provided (4.28) holds. Therefore, $w_{n} \in L^{\infty}(0, T ; D(A))$. From (4.27), we obtain that each $w_{n}$ belongs to a bounded set of $L^{2}\left(0, T ; D\left(A^{3 / 2}\right)\right)$ for any $T$. Therefore, as usual choosing a subsequence that converges weakly in $L^{2}$ and weakly* in $L^{\infty}$, and taking the limit as $n \rightarrow \infty$, we obtain the solution of (4.7). Note that (4.28) implies (4.24). Therefore, the following lemma is true.

Lemma 4.2: Suppose $h \in D\left(A^{1 / 2}\right)$. If $G$ and $N$ satisfy (4.28), then the solution to (4.7) satisfies

$$
|A w| \leq \frac{\|h\|+9 v^{2} \kappa_{0}^{3} G^{2} N\left(c_{T}(\ln e N)^{1 / 2}+c_{\mathrm{L}}\right)}{\nu \kappa_{0}\left\{N-c_{\mathrm{L}} G(9+\beta(G, N))\right\}} .
$$

We note that the analog of the results in this section with $w \in C_{b}\left((-\infty, T], Q_{N} H\right)$ instead of $C_{b}\left(\mathbb{R}, Q_{N} H\right)$ in this section remains valid for $v \in C_{b}\left((-\infty, T], P_{N} H\right)$.

\section{B. Lipschitz property of $W$}

Let $v_{1}, v_{2}$ be in $X$, and $w_{1}=W\left(v_{1}\right), w_{2}=W\left(v_{2}\right)$. Denote $\gamma=w_{1}-w_{2}, \delta=v_{1}-v_{2}$, $\tilde{v}_{i}=\varphi\left(\frac{\left\|v_{i}\right\|}{\nu \kappa_{0}}\right) v_{i}, i=1,2, \tilde{\delta}=\tilde{v}_{1}-\tilde{v}_{2}$. Subtracting Eq. (4.7) for $w_{2}$ from that for $w_{1}$, we obtain

$$
\frac{d}{d s} \gamma+v A \gamma+Q_{N} B\left(\tilde{\delta}+\gamma, \tilde{v}_{1}+w_{1}\right)+Q_{N} B\left(\tilde{v}_{2}+w_{2}, \tilde{\delta}+\gamma\right)=0 .
$$

Taking the scalar product with $A \gamma$, we have

$$
\begin{aligned}
& \left.\frac{1}{2} \frac{d}{d s}\|\gamma\|^{2}+v|A \gamma|^{2} \leq\left|\left(B\left(\tilde{\delta}, \tilde{v}_{1}+w_{1}\right), A \gamma\right)\right|+\mid B\left(\tilde{v}_{2}+w_{2}, \tilde{\delta}\right), A \gamma\right) \mid \\
& \left.\left.\quad+\left|\left(B\left(\gamma, \tilde{v}_{1}\right), A \gamma\right)\right|+\mid B\left(\tilde{v}_{2}, \gamma\right), A \gamma\right)|+|\left(B\left(\gamma, w_{1}\right), A \gamma\right)|+| B\left(w_{2}, \gamma\right), A \gamma\right) \mid .
\end{aligned}
$$


To estimate the right-hand side we apply (A19) to the first term, (A20) to the second, (A22) to the third term, (A2), (A4), and (A24) to the fourth term, and (A23) and (A22), respectively, to the fifth and sixth terms. Using then (4.10), (4.18), and (4.22), we have

$$
\begin{aligned}
\frac{1}{2} \frac{d}{d s}\|\gamma\|^{2} & +v|A \gamma|^{2} \leq G(3+\beta(G, N)) \kappa_{0} v\left(c_{T}+c_{B}\right)\|\tilde{\delta}\||A \gamma|(\ln e N)^{1 / 2} \\
& +3 v\left(c_{\mathrm{A}}+c_{\mathrm{L}}\right)|A \gamma|^{2} \frac{G}{N}+v\left(c_{\mathrm{A}}+c_{\mathrm{L}}\right) \frac{G \beta(G, N)}{N}|A \gamma|^{2},
\end{aligned}
$$

and thus by Lemma 3.2 (ii), we have that

$$
\|\gamma(s)\| \leq \frac{G(3+\beta(G, N))(\ln e N)^{1 / 2}\left(c_{T}+c_{B}\right)}{N-G(3+\beta(G, N))\left(c_{\mathrm{A}}+c_{\mathrm{L}}\right)} \sup _{s^{\prime} \leq s}\left\|\tilde{\delta}\left(s^{\prime}\right)\right\|
$$

for $G, N$ such that

$$
N>G(3+\beta(G, N))\left(c_{\mathrm{A}}+c_{\mathrm{L}}\right) .
$$

From now on, we will consider $G$ and $N$ such that

$$
N>G(9+\beta(G, N))\left(c_{\mathrm{A}}+c_{\mathrm{L}}\right),
$$

which implies (4.12), (4.24), (4.35), and (4.28).

We derive from (4.34) the following two corollaries.

Corollary 4.3: Suppose that $G$ and $N$ satisfy (4.36). Then if $v_{1}, v_{2} \in X, v_{1}(s)=v_{2}(s)$ for all $s \leq s_{0}$ we have that $W\left(s, v_{1}\right)=W\left(s, v_{2}\right)$ for all $s \leq s_{0}$.

Proof: Indeed, if $s \leq s_{0}$, it follows that $\tilde{\delta}(\sigma)=0$ for all $\sigma \leq s$, thus (4.34) gives that $\gamma(s)=0$.

Corollary 4.4: Suppose that $G$ and $N$ satisfy (4.36). Then $W: X \rightarrow Y$ is a Lipschitz function with Lipschitz constant $L_{W}$ satisfying

$$
L_{W} \leq 4 \frac{G(3+\beta(G, N))(\ln e N)^{1 / 2}\left(c_{T}+c_{B}\right)}{N-G(3+\beta(G, N))\left(c_{\mathrm{A}}+c_{\mathrm{L}}\right)} .
$$

Proof: If we have both $\left\|v_{1}\right\| \geq 3 v \kappa_{0} G$ and $\left\|v_{2}\right\| \geq 3 G$, then $\tilde{\delta}=0$. If, say $\left\|v_{1}\right\| \leq 3 G$, then

$$
\begin{aligned}
\left\|\tilde{v}_{1}-\tilde{v}_{2}\right\| & \leq\left|\varphi\left(\frac{\left\|v_{1}\right\|}{v \kappa_{0}}\right)-\varphi\left(\frac{\left\|v_{2}\right\|}{v \kappa_{0}}\right)\right|\left\|v_{1}\right\|+\varphi\left(\frac{\left\|v_{2}\right\|}{\nu \kappa_{0}}\right)\left\|v_{1}-v_{2}\right\| \\
& \leq \frac{1}{v \kappa_{0} G}\left|\left\|v_{1}\right\|-\left\|v_{2}\right\|\right| 3 \nu \kappa_{0} G+\left\|v_{1}-v_{2}\right\|
\end{aligned}
$$

thus

$$
\|\tilde{\delta}\| \leq 4\|\delta\|
$$

Theorem 4.5: Suppose $G$ and $N$ satisfy (4.36). Equation (2.17) is an ordinary differential equation in the Banach space $C_{b}\left(\mathbb{R}, P_{N} H\right)$.

Proof: It suffices to show that $F(v)=P_{N} f-v A v-P_{N} B(v+W(v), v+W(v))$ is Lipschitz. To estimate $L_{F}$, the Lipschitz constant of $F$, we apply (A18), (4.10), and (4.18) and Corollary 4.4 to 
obtain for every (nonspecified) $s \in \mathbb{R}$,

$$
\begin{aligned}
& \eta(s)=\left|P_{N} B\left(\tilde{v}_{1}+W\left(v_{1}\right), \tilde{v}_{1}+W\left(v_{1}\right)\right)-P_{N} B\left(\tilde{v}_{2}+W\left(v_{2}\right), \tilde{v}_{2}+W\left(v_{2}\right)\right)\right| \\
& \leq \mid P_{N} B\left(\tilde{v}_{1}+W\left(v_{1}\right), \tilde{v}_{1}+W\left(v_{1}\right)\right)-P_{N} B\left(\tilde{v}_{1}+W\left(v_{1}\right), \tilde{v}_{2}+W\left(v_{2}\right)\right) \mid \\
&+\left|P_{N} B\left(\tilde{v}_{1}+W\left(v_{1}\right), \tilde{v}_{2}+W\left(v_{2}\right)\right)-P_{N} B\left(\tilde{v}_{2}+W\left(v_{2}\right), \tilde{v}_{2}+W\left(v_{2}\right)\right)\right| \\
&=\left|P_{N} B\left(\tilde{v}_{1}+W\left(v_{1}\right), \tilde{v}_{1}-\tilde{v}_{2}+W\left(v_{1}\right)-W\left(v_{2}\right)\right)\right| \\
&+\left|P_{N} B\left(\tilde{v}_{1}-\tilde{v}_{2}+W\left(v_{1}\right)-W\left(v_{2}\right), \tilde{v}_{2}+W\left(v_{2}\right)\right)\right| \\
& \leq c_{T}\left(\left\|\tilde{v}_{1}+W\left(v_{1}\right)\right\|+\left\|\tilde{v}_{2}+W\left(v_{2}\right)\right\|\right)(\ln e N)^{1 / 2}\left\|\tilde{v}_{1}-\tilde{v}_{2}+W\left(v_{1}\right)-W\left(v_{2}\right)\right\| .
\end{aligned}
$$

It follows that

$$
\sup _{s \in \mathbb{R}} \eta(s) \leq 2 c_{T} v \kappa_{0} G(3+\beta(G, N))(\ln e N)^{1 / 2}\left(3+L_{W}\right)\left\|v_{1}-v_{2}\right\|_{X}
$$

and, therefore,

$$
\begin{aligned}
& \left\|F\left(v_{1}\right)-F\left(v_{2}\right)\right\|_{X} \leq \kappa_{0} N \sup _{s \in \mathbb{R}}\left|F\left(v_{1}(s)\right)-F\left(v_{2}(s)\right)\right| \\
& \quad \leq\left[\nu \kappa_{0}^{2} N^{2}+2 c_{T} \nu \kappa_{0}^{2} N G(3+\beta(G, N))(\ln e N)^{1 / 2}\left(3+L_{W}\right)\right]\left\|v_{1}-v_{2}\right\|_{X} .
\end{aligned}
$$

We denote the solution operator for (2.17) by $S_{\mathrm{DF}}$, i.e., $S_{\mathrm{DF}}(t) v_{0}=v(t)$, where $v(t)$ is the solution with initial value $v(0)=v_{0}$. Using (4.26), we obtain the following corollary which establishes Proposition 2.5 .

Corollary 4.6: $\tau_{\theta} S_{\mathrm{DF}}(t)=S_{\mathrm{DF}}(t) \tau_{\theta}$.

Recall that for $v(t) \in X$ we denote by $v(t, s)$ the value of $v(t)$ at the parameter $s \in \mathbb{R}$.

Lemma 4.7: Suppose $v$ is a solution of (2.17) such that $\|v(0)\|_{X} \leq 2 v \kappa_{0} G$ and

$$
v(t, s)=v(0, t+s) \text { for all } t, s \in \mathbb{R} .
$$

Then $u(t)=v(0, t)+W(t, v(0, \cdot))$ is the solution of $(2.1)$ satisfying

$$
P_{n} u(t)=v(0, t)=v(t, 0) \quad \text { for all } t \in \mathbb{R} .
$$

Proof: We first note that by (4.39) and (4.26),

$$
W(0 ; v(t, \cdot))=W\left(0 ; \tau_{t} v(0, \cdot)\right)=\tau_{t} W(0 ; v(0, \cdot))=W(t ; v(0, \cdot)),
$$

so we can set

$$
w(t)=W(0 ; v(t))=W(t ; v(0)) .
$$

Since $\|v(0)\| \leq 2 v \kappa_{0} G$ we have $\varphi=1$, where $\varphi$ is as in (2.18). By (4.39) and (4.40), and the definition of the map $W$, we have

$$
\begin{aligned}
\frac{d}{d t} w(t)+v A w(t)+Q_{N} B(v(0, t)+w(t)) & = \\
\frac{d}{d t} w(t)+v A w(t)+Q_{N} B(v(t, 0)+W(t ; v(0, \cdot))) & =Q_{N} f
\end{aligned}
$$

as well as

$$
\begin{aligned}
\frac{d}{d t} v(t)+v A v(t)+P_{N} B(v(t)+w(t)) & = \\
\frac{d}{d t} v(t, 0)+v A v(t, 0)+P_{N} B(v(t, 0)+W(0 ; v(t, \cdot))) & =P_{N} f .
\end{aligned}
$$

Adding Eqs. (4.42) and (4.41), we see that $u(t)=v(0, t)+W(t, v(0, \cdot))$ is a solution of (2.1). 
We also have the following property of (2.17).

Proposition 4.8: Suppose that $v_{1}(0, s)=v_{2}(0, s)$ for $s \leq s_{0}$. Then $v_{1}(t, s)=v_{2}(t, s)$ for $s \leq s_{0}$ for any $t$.

Proof: By Corollary 4.3, the map $W$ is well-defined on the space $C_{b}\left(\left(-\infty, s_{0}\right], P_{N} H\right)$. Thus, we can consider the differential equation (2.17) on this space. It is still an ordinary differential equation, and so through each point there is only one solution. Each solution of (2.17) on the whole $X$ gives a solution of (2.17) on $C_{b}\left(\left(-\infty, s_{0}\right], P_{N} H\right)$, by restriction. Since solutions $v_{1}(t)$ and $v_{2}(t)$ of the restriction of (2.17) on $C_{b}\left(\left(-\infty, s_{0}\right], P_{N} H\right)$ coincide for $t=0$, they coincide for all $t$.

\section{ABSORBING BALL}

We will now prove that the determining form (2.17) has an absorbing ball in $X$. Recall that this means an estimate of the type (A14) holds but with $\|\cdot\|$ replaced by $|\cdot|_{X}$. Let $g=P_{N} f$ and take the scalar product with $v=v(t, s)$ (and denote $W(v)$ by $w$ ) to obtain

$$
\begin{aligned}
& \frac{1}{2} \frac{d}{d t}|v|^{2}+v\|v\|^{2} \leq|g \| v|+|\varphi(B(v, v), w)|+|\varphi(B(w, v), w)| \\
& \quad \leq|g|\|v\|+c_{B} \varphi\|v\|^{2}|w|(\ln e N)^{1 / 2}+c_{\mathrm{L}} \varphi|w|\|w\|\|v\| \\
& \quad \leq \kappa_{0} v^{2} \frac{|g|}{|f|} G\|v\|+v c_{B}\|v\|^{2} \frac{G \beta(G, N)}{N}(\ln e N)^{1 / 2}+\kappa_{0} v^{2} c_{\mathrm{L}} \frac{G^{2} \beta(G, N)^{2}}{N}\|v\|,
\end{aligned}
$$

where we used inequalities (A19), (A21), (4.18), and (4.22). It follows from Lemma 3.1 that if

$$
N>c_{B} G \beta(G, N)(\ln e N)^{1 / 2}
$$

holds, we have the following.

Proposition 5.1: If (5.1) and (4.36) hold, then

$$
|v| \leq v G \frac{\frac{|g|}{|f|}+c_{\mathrm{L}} G \frac{\beta(G, N)^{2}}{N}}{1-c_{B} \frac{G \beta(G, N)}{N}(\ln e N)^{1 / 2}} .
$$

While the relation $\|v\| \leq \kappa_{0} N|v|$ provides an immediate bound on $\|v\|$ provided (5.1) holds, we can derive a sharper bound on $\|v\|$. As above, taking the scalar product with $A v$ we have

$$
\begin{aligned}
\frac{1}{2} \frac{d}{d t}\|v\|^{2}+v|A v|^{2} & =(g, A v)-(B(\tilde{v}+w, \tilde{v}+w), A v) \\
& \leq|g||A v|-(B(\tilde{v}, w), A v)-(B(w, \tilde{v}), A v)-(B(w, w), A v) \\
& \leq|g||A v|+c_{T}\left(2\|\tilde{v}\|\|w\||A v|+\|w\|^{2}|A v|\right)(\ln e N)^{1 / 2} \\
& \leq v^{2} \kappa_{0}^{2}\left[\frac{|g|}{|f|} G+c_{T}(\ln e N)^{1 / 2} G^{2}(6+\beta(G, N)) \beta(G, N)\right]|A v|,
\end{aligned}
$$

where $v=v(t, s)$ and (5.3) is valid at each $s \in \mathbb{R}$. Applying Lemma 3.1 (i) and then (ii) shows that the ball centered at the origin, with radius (strictly) greater than

$$
r_{0}=v \kappa_{0} G\left[\frac{|g|}{|f|}+c_{T}(\ln e N)^{1 / 2} G(6+\beta(G, N)) \beta(G, N)\right]
$$

is an absorbing ball in $X$ for (2.17), which completes the proof of Theorem 2.3.

Remark 5.1: The $O\left(G^{2}\right)$ estimate (up to a logarithm) for the radius of the absorbing ball for (2.17) in (5.4) is significantly larger than the $O(G)$ estimate for radius of the absorbing ball for the 
NSE. These estimates can be brought closer if we assume $N$ is larger. Indeed, if we take for some $\gamma>1$,

$$
N \geq\left(6 c_{T} G(\ln e N)^{1 / 2}\right)^{\gamma}
$$

then

$$
\beta(G, N) \leq \frac{9 N^{1 / \gamma} /\left(6 c_{T}\right)+1}{N-N^{\frac{1}{\gamma}} / 2} \leq \frac{3 N^{\frac{1}{\gamma}}+2}{N} \leq 5 N^{\frac{1-\gamma}{\gamma}},
$$

and consequently

$$
G(\ln e N)^{1 / 2} \beta(G, N) \leq \frac{5}{6 c_{T}} N^{\frac{2-\gamma}{\gamma}} .
$$

Thus, if equality holds in (5.5) with $\gamma=3 / 2$, we have (up to a logarithm) that $r_{0}=O\left(G^{3 / 2}\right.$ ), and if (5.5) holds with $\gamma=2$, we have $r_{0}=O(G)$.

Let $\mathcal{B}_{r}$ be an absorbing ball in $X$, centered at 0 with radius $r>r_{0}$, and let $\mathcal{M}=\cap_{t>0} S_{\mathrm{DF}}(t) \mathcal{B}_{r}$. Note that $\mathcal{M}$ is independent of $r: v_{0} \in X$ belongs to $\mathcal{M}$ if and only if there is a bounded solution $v$ : $(-\infty, \infty) \rightarrow X$ of (4.5) defined on the whole real line such that $v(0)=v_{0}$. We can also characterize $\mathcal{M}$ as the maximal bounded set that is invariant (i.e., $S_{\mathrm{DF}}(t) \mathcal{M}=\mathcal{M} \forall t \geq 0$ ), both forward and backward in time, under the flow generated by (2.17). We do not know if $\mathcal{M}$ is the analog for the determining form (2.17) of the global attractor of the Navier-Stokes equations (see discussion leading to (2.8)).

By Theorem 2.3, $\mathcal{M}$ is a subset of $\mathcal{B}_{r_{0}}$.

Lemma 5.2: If $v$ is a bounded solution of (4.5) defined on the whole real line and $\|W(v(t))\|_{Y} \leq v \kappa_{0} \sqrt{2 G / c_{L}}$ for all $t$, then $\|v(t)\|_{X} \leq 3 v \kappa_{0} G$ for all $t$. In particular, this is true if $N \geq \sqrt{2 c_{\mathrm{L}}}\left(9 c_{T} G^{3 / 2}(\ln e N)^{1 / 2}+G^{1 / 2}\right)$ and $N \geq 6 c_{\mathrm{L}} G$.

Proof: Let $w(t)=W(v(t))$ and $w(t, s)=w(t)(s)$. Suppose that $\left\|v\left(t^{\prime}, s^{\prime}\right)\right\|>3 v \kappa_{0} G$ for some $t^{\prime}$ and $s^{\prime}$. Then since $v$ is continuous there are $t_{0}<t^{\prime}<t_{1}$ such that in the interval $\left[t_{0}, t_{1}\right]$ we have $\left\|v\left(t, s^{\prime}\right)\right\| \geq 3 v \kappa_{0} G$. On this interval, the map $t \mapsto v\left(t, s^{\prime}\right)$ satisfies the equation

$$
\frac{d}{d t} v\left(t, s^{\prime}\right)+v A v\left(t, s^{\prime}\right)+B\left(w\left(t, s^{\prime}\right)\right)=g .
$$

Taking its scalar product with $A v\left(t, s^{\prime}\right)$, we obtain inequality

$$
\frac{d}{d t}\left\|v\left(t, s^{\prime}\right)\right\|^{2}+v\left|A v\left(t, s^{\prime}\right)\right|^{2} \leq|g|\left|A v\left(t, s^{\prime}\right)\right|+\left|\left(B\left(w\left(t, s^{\prime}\right), A v\left(t, s^{\prime}\right)\right), w\left(t, s^{\prime}\right)\right)\right|
$$

and using (A21) we have that $|(B(w, A v), w)| \leq c_{\mathrm{L}}|w|\|w\|\left|A^{3 / 2} v\right| \leq c_{\mathrm{L}}\|w\|_{Y}^{2}|A v|$, and hence

$$
\frac{d}{d t}\left\|v\left(t, s^{\prime}\right)\right\|^{2}+v\left|A v\left(t, s^{\prime}\right)\right|^{2} \leq\left(G v^{2} \kappa_{0}^{2}+c_{\mathrm{L}}\|w\|_{Y}^{2}\right)\left|A v\left(t, s^{\prime}\right)\right| .
$$

Note that by Lemma 3.1 (i) $t_{0}=-\infty$, since $G v \kappa_{0}+c_{\mathrm{L}}\|w(t)\|^{2} /\left(v \kappa_{0}\right)<3 G v \kappa_{0}$, but by the Lemma 3.1 (iii) this is impossible.

Remark 5.2: It is easy to give an analytic characterization of the solutions of (2.17) in $\mathcal{M}$. Indeed we have

$$
v(t)=e^{-v\left(t-t_{0}\right) A} v\left(t_{0}\right)+\int_{t_{0}}^{t} e^{-v(t-\theta) A}\left[g-P_{N} B(\tilde{v}(\theta)+W(v(\theta)))\right] d \theta,
$$

and letting $t_{0} \rightarrow-\infty$ we easily obtain the relation

$$
v(t)=\int_{-\infty}^{t} e^{-v(t-\theta) A}\left[g-P_{N} B(\tilde{v}(\theta)+W(v(\theta)))\right] d \theta \quad t>0,
$$

in X. Clearly, (5.6) implies that $v(t)$ is in $\mathcal{M}$. 
In the case, when

$$
v(t, s)=P_{N} u(t+s), \quad s, t \in \mathbb{R},
$$

where $u(t)$ is a solution of (2.1) in $\mathcal{A}$, it is easy to see that the relation (5.6) is equivalent to

$$
v(s)=\int_{-\infty}^{s} e^{-v(s-\sigma) A}\left[g-P_{N} B(\tilde{v}(\sigma)+W(v(\sigma)))\right] d \sigma \quad s \in \mathbb{R} .
$$

It follows that

$$
v(s)=P_{N} u(s), \quad s \in \mathbb{R},
$$

where $u(s)$ is a solution of (2.1) in $\mathcal{A}$ if and only if $v \in X$ and satisfies (in $H$ ) the relation (5.7).

\section{TRAVELING WAVES}

We say that $v(t, s)=S_{\mathrm{DF}}(t) v_{0}(s)$ is a traveling wave solution for (2.17) if

$$
v(t, s)=v_{0}(t+s) .
$$

Denote $w(t, s)=W(s ; v(t, \cdot)), u(t, s)=v(t, s)+w(t, s)$, and define $w_{0} \in Y, u_{0} \in C_{b}(\mathbb{R}, H)$ by $w_{0}(s)=w(0, s), u_{0}(s)=u(0, s)$. Suppose $v$ is a traveling wave solution. Then by (6.1) and (4.26) $w(t, s)=w_{0}(t+s)$, thus $u(t, s)=u_{0}(t+s)$. We will provide an equation for $u_{0}$ and use it to derive the properties of translation invariant solutions. Using (6.1), Eq. (2.17) can be written as

$$
\frac{d}{d t} v_{0}+v A v_{0}=P_{N} f-P_{N} B\left(\varphi v_{0}+w_{0}\right)
$$

while (4.7) is written as

$$
\frac{d}{d s} w_{0}+v A w_{0}=Q_{N} f-Q_{N} B\left(\varphi v_{0}+w_{0}\right)
$$

Changing $t$ to $s$ in (6.2) and combining the result with (6.3), we have the equation for $u_{0}$,

$$
\frac{d}{d s} u_{0}+v A u_{0}=f-B\left(\varphi v_{0}+w_{0}\right) .
$$

Taking the scalar product with $A u_{0}$, we have

$$
\frac{1}{2} \frac{d}{d s}\left\|u_{0}\right\|^{2}+v\left|A u_{0}\right|^{2}=\left(f, A u_{0}\right)-\left(B\left(\varphi v_{0}+w_{0}\right), A u_{0}\right) .
$$

Using (A5) and (A3), we obtain

$$
\begin{gathered}
\left(B\left(\varphi v_{0}+w_{0}\right), A u_{0}\right)=\varphi\left[\left(B\left(v_{0}, w_{0}\right), A v_{0}\right)+\left(B\left(w_{0}, v_{0}\right), A v_{0}\right)\right]+\left(B\left(w_{0}, w_{0}\right), A v_{0}\right) \\
+\varphi^{2}\left(B\left(v_{0}, v_{0}\right), A w_{0}\right)+\varphi\left[\left(B\left(v_{0}, w_{0}\right), A w_{0}\right)+\left(B\left(w_{0}, v_{0}\right), A w_{0}\right)\right] \\
=\left(\varphi^{2}-\varphi\right)\left(B\left(v_{0}, v_{0}\right), A w_{0}\right)+(1-\varphi)\left(B\left(w_{0}, w_{0}\right), A v_{0}\right)
\end{gathered}
$$

so by applying (A5) again, we have

$$
\begin{aligned}
\mid\left(B \left(\varphi v_{0}\right.\right. & \left.\left.+w_{0}\right), A u_{0}\right)|\leq \varphi|\left(B\left(v_{0}, v_{0}\right), A w_{0}\right)|+|\left(B\left(w_{0}, w_{0}\right), A v_{0}\right) \mid \\
& \leq \varphi\left|\left(B\left(v_{0}, w_{0}\right), A v_{0}\right)\right|+\varphi\left|\left(B\left(w_{0}, v_{0}\right), A v_{0}\right)\right|+\left|\left(B\left(w_{0}, w_{0}\right), A v_{0}\right)\right|
\end{aligned}
$$

We now apply (A18), (4.10), and (4.25) to obtain

$$
\begin{aligned}
\frac{1}{2} \frac{d}{d s}\left\|u_{0}\right\|^{2}+v\left|A u_{0}\right|^{2} & \leq\left|f \| A u_{0}\right|+c_{T}(6+\beta(G, N)) v^{2} \kappa_{0}^{2} G^{2} \beta(G, N)(\ln e N)^{1 / 2}\left|A v_{0}\right| \\
& \leq v^{2} \kappa_{0}^{2} G\left[1+c_{T}(6+\beta(G, N)) G \beta(G, N)(\ln e N)^{1 / 2}\right]\left|A u_{0}\right|
\end{aligned}
$$

and consequently by Lemma 3.1 (iii),

$$
\left\|u_{0}(s)\right\| \leq \nu \kappa_{0} G\left[1+c_{T}(6+\beta(G, N)) G \beta(G, N)(\ln e N)^{1 / 2}\right] .
$$


We next calculate an absolute constant $c_{T}^{\prime}$ such that if

$$
N \geq c_{T}^{\prime} G \beta(G, N) \ln e N,
$$

then

$$
c_{T}(6+\beta(G, N)) G \beta(G, N)(\ln e N)^{1 / 2} \leq 1,
$$

so that (6.6) implies

$$
\left\|v_{0}(s)\right\| \leq\left\|u_{0}(s)\right\| \leq 2 v \kappa_{0} G .
$$

First note that for $c_{T}^{\prime} \geq 18 c_{T}$, we have $\beta(G, N) \leq 2$, for all $N \geq 1$. Then estimate

$$
\begin{aligned}
8 c_{T} G \beta(G, N)(\ln e N)^{1 / 2} & =8 c_{T} \frac{9 c_{T} G \ln e N+(\ln e N)^{1 / 2}|h| /|f|}{N-3 c_{T} G(\ln e N)^{1 / 2}} \\
& \leq 8 c_{T} \frac{9 c_{T} N / c_{T}^{\prime}+N / c_{T}^{\prime}}{N-3 c_{T} N / c_{T}^{\prime}}=\frac{72 c_{T}^{2}+8 c_{T}}{c_{T}^{\prime}-3 c_{T}},
\end{aligned}
$$

so that it suffices in (6.7) to take

$$
c_{T}^{\prime} \geq 72 c_{T}^{2}+11 c_{T} .
$$

Proof of Theorem 2.4: Suppose (6.7) holds for $c_{T}^{\prime}$ as in (6.9). The fact that any traveling wave solution of (2.17) is the projection of a solution of (2.1) follows from (6.8) and Lemma 4.7. To prove the converse, recall that any solution $u(t)$ of the (2.1) which lies on the attractor can be extended to a solution to the complexified NSE which is analytic and bounded on some strip $|\mathfrak{J} t|<c$ function into $H$ (see, e.g., Ref. 6). Let $u(t)$ be a solution of (2.1) defined and bounded on the whole real line. Consider a curve in $X, t \mapsto(s \mapsto \mathfrak{p}(t+s))$, where $\mathfrak{p}=P_{N} u$. We need to show that this curve is differentiable and its derivative is $s \mapsto d \mathfrak{p} / d t(t+s)$; thus we need to prove that for any real $t$,

$$
\sup _{s \in \mathbb{R}}\left\|\frac{\mathfrak{p}(t+\Delta t+s)-\mathfrak{p}(t+s)}{\Delta t}-\frac{d}{d t} \mathfrak{p}(t+s)\right\| \rightarrow 0 \quad \text { as } \quad \Delta t \rightarrow 0 .
$$

Since $\mathfrak{p}$ is analytic and bounded in the strip $|\mathfrak{J} \zeta|<c$, its second derivative is also bounded in a smaller strip. The limit in (6.10) now follows from Taylor's theorem, specifically

$$
\sup _{s \in \mathbb{R}}\left\|\frac{\mathfrak{p}(t+\Delta t+s)-\mathfrak{p}(t+s)}{\Delta t}-\frac{d}{d t} \mathfrak{p}(t+s)\right\| \leq \sup _{s \in \mathbb{R}} \Delta t \sup _{\tau \in[t, t+t+\Delta t]}\left\|\frac{d^{2}}{d t^{2}} \mathfrak{p}(\tau+s)\right\| .
$$

\section{EQUICONTINUITY OF $w$}

We have from (4.11) and (4.20) that

$$
\int_{s_{0}}^{s_{1}}|A w|^{2} \leq E_{1}\left(s_{1}-s_{0}\right)+E_{0}
$$

for some constants $E_{0}, E_{1}$. We also have for any $u \in H$,

$$
\begin{aligned}
|(B(\varphi v+w), u)| \leq 9 c_{B} G^{2} v^{2} \kappa_{0}^{2}(\ln e N)^{1 / 2}|u| & +3\left(c_{\mathrm{A}}+c_{T}\right) v^{2} \kappa_{0}^{2} G^{2} \beta(G, N)(\ln e N)^{1 / 2}|u| \\
& +c_{\mathrm{A}} v \kappa_{0} \frac{G}{N} \beta(G, N)|A w||u| ;
\end{aligned}
$$

thus

$$
|B(\varphi v+w)| \leq E_{2}+E_{3}|A w|,
$$


where

$$
\begin{aligned}
& E_{2}=\left[9 c_{B}+3\left(c_{B}+c_{T}\right) \beta(G, N)\right] v^{2} \kappa_{0}^{2} G^{2} \beta(G, N)(\ln e N)^{1 / 2}, \\
& E_{3}=c_{\mathrm{A}} v \kappa_{0} \frac{G}{N} \beta(G, N) .
\end{aligned}
$$

Integrating (4.7) from $s_{0}$ to $s_{1}$ we have

$$
\begin{aligned}
\left|w\left(s_{1}\right)-w\left(s_{0}\right)\right| & \leq v \int_{s_{0}}^{s_{1}}|A w|+\int_{s_{0}}^{s_{1}}|B(\varphi v+w, \varphi v+w)|+\int_{s_{0}}^{s_{1}}|h| \\
& \leq\left(v+E_{3}\right)\left(s_{1}-s_{0}\right)^{1 / 2}\left[E_{1}\left(s_{1}-s_{0}\right)+E_{0}\right]^{1 / 2}+\left(E_{2}+|h|\right)\left(s_{1}-s_{0}\right) .
\end{aligned}
$$

We have proved the following.

Lemma 7.1: The family $\left\{W(v)(\cdot) \mid v \in C_{b}\left(\mathbb{R}, P_{N} H\right)\right\}$ is equicontinuous on $\mathbb{R}$.

Let $v(t)$ be a solution of (2.17), and denote $w(s, t)=W(v(t))(s)$. Then by Lemma 4.4,

$$
\left\|w(s, t)-w\left(s, t_{0}\right)\right\| \leq L_{W}\left\|v(t)-v\left(t_{0}\right)\right\|_{X} .
$$

Since $v(t)$ is a solution of an ODE, it is continuous. Thus for any finite interval $[-T, T]$ there is a constant $C_{T}$, such that

$$
\left\|v(t)-v\left(t_{0}\right)\right\|_{X} \leq C_{T}\left|t-t_{0}\right|, \quad t, t_{0} \in[-T, T] .
$$

This proves the following.

Proposition 7.2: For any solution $v(t)$ of Eq. (2.17) the function $w(s, t)=W(v(t))(s)$ is equicontinuous on $\mathbb{R}^{2}$. Moreover, there are constants $c, c_{1}$, and $C_{T}$ such that

$$
\left|w(s, t)-w\left(s_{0}, t_{0}\right)\right| \leq c\left|s-s_{0}\right|^{1 / 2}+c_{1}\left|s-s_{0}\right|+\frac{C_{T}}{N}\left|t-t_{0}\right|,
$$

whenever $t, t_{0} \in[-T, T]$.

We now assume that (4.36) holds, and that $h \in D\left(A^{1 / 2}\right)$. Then by (4.30), we have

$$
|A W(v)(s)| \leq \Gamma ; \quad \text { for all } v \in X, s \in \mathbb{R},
$$

where $\Gamma$ is the right-hand side of (4.30). We have, therefore, that

$$
\begin{aligned}
& \left\|w(s, t)-w\left(s_{0}, t_{0}\right)\right\| \leq(2 \Gamma)^{1 / 2}\left|w(s, t)-w\left(s_{0}, t_{0}\right)\right|^{1 / 2} \\
& \quad \leq(2 \Gamma)^{1 / 2}\left[c\left|s-s_{0}\right|^{1 / 2}+c_{1}\left|s-s_{0}\right|+\frac{C_{T}}{N}\left|t-t_{0}\right|\right]^{1 / 2}
\end{aligned}
$$

for all $s_{0}, s_{1} \in \mathbb{R}$, and all $t, t_{0} \in[-T, T]$.

Recall the extension of the Arzela-Ascoli theorem to the case of Banach-valued functions.

Theorem 7.3: Let $K$ be a compact metric space, $\mathcal{X}$ a Banach space, $\Phi_{n}: k \mapsto \Phi_{n}(k) \in \mathcal{X}$, with $\left\{\Phi_{n}\right\}_{n=1}^{\infty}$ equicontinuous and $\left\{\Phi_{n}(k)\right\}_{n=1}^{\infty}$ relatively compact for each $k \in K$. Then there exists a subsequence $\left\{\Phi_{n_{j}}\right\}_{j=1}^{\infty}$ which converges in $C(K ; \mathcal{X})$.

\section{WEAK LIMIT OF TIME TRANSLATED SOLUTIONS}

According to Remark 2.1 (Sec. VII), a solution $v$ to the determining form (2.17) in the set $\mathcal{M}$ is the $P_{N}$-projection of a solution of the Navier-Stokes Equation (2.1) in its global attractor $\mathcal{A}$ if and only if

$$
\|v(t, s)\| \leq 2 v \kappa_{0}^{2} G, \quad \forall t, s \in \mathbb{R}
$$

and

$$
v\left(t+t_{n}, s-\left(t+t_{n}\right)\right)=v(0, s), \quad \forall t, s \in \mathbb{R},
$$


for any sequence $\left\{t_{n}\right\}$. It is, therefore, instructive to study the asymptotic behavior of a sequence

$$
v_{n}(t, s)=v\left(t+t_{n}, s-\left(t+t_{n}\right)\right), \quad n=1,2, \ldots,
$$

where $t_{n} \rightarrow \infty$, and $v(\cdot) \in \mathcal{M}$ is a solution of (2.17) satisfying (8.1). We have the following lemma.

Lemma 8.1: There is a subsequence $\left\{n_{j}\right\}$ and functions $\bar{v}, \bar{w}$, and $\bar{R}$ such that $\bar{v}$, $\bar{R} \in C\left(\mathbb{R}, L^{\infty}(\mathbb{R}, H)_{\text {weak }^{*}}\right), \bar{w} \in C\left(\mathbb{R}^{2}, V\right)$ and the following hold for each $T, S:$

$$
\begin{gathered}
\lim _{j} \sup _{t \in[-T, T]}\left|\left(v_{n_{j}}(t, \cdot)-\bar{v}(t, \cdot), \xi\right)\right|=0 \quad \text { for } \xi \in L^{1}(\mathbb{R}, H), \\
\lim _{j} \sup _{t \in[-T, T]}\left|\left(B\left(v_{n_{j}}(t, \cdot)-\bar{v}(t, \cdot)\right)+\bar{R}(t, \cdot), \xi\right)\right|=0 \quad \text { for } \xi \in L^{1}(\mathbb{R}, H), \\
w_{n_{j}}(t, s)=W\left(\tau_{t_{n_{j}}} v\right)\left(s-\left(t+t_{n_{j}}\right)\right) \rightarrow \bar{w}(t, s) \quad(t, s \in \mathbb{R})
\end{gathered}
$$

in $C([-T, T] \times[-S, S] ; V)$, for all $T, S>0$

Proof: It follows from (2.17) and (7.3) that there exists a constant $C$,

$$
\left|\frac{\partial v_{n}(t, s)}{\partial t}\right| \leq C \quad(\forall t, s, n) .
$$

Choose a dense subset $\left\{\xi_{k}\right\}$ in the unit ball of $L^{1}(\mathbb{R}, H)$. Then it is well-known and easy to check that

$$
d\left(p, p^{\prime}\right)=\sum_{k} \frac{\left|\left(p-p^{\prime}, \xi_{k}\right)\right|}{2^{k}\left(1+\left|\left(p-p^{\prime}, \xi_{k}\right)\right|\right)}
$$

defines a distance in the ball of any fixed size in $L^{\infty}(\mathbb{R}, H)$. Let us compute $d\left(v_{n}(t, \cdot), v_{n}\left(t^{\prime}, \cdot\right)\right)$. Note first that

$$
\begin{aligned}
& \left|\int_{\mathbb{R}}\left(v_{n}(t, s)-v_{n}\left(t^{\prime}, s\right), \xi_{k}(s)\right) d s\right| \\
& \quad=\left|\int_{\mathbb{R}}\left(v\left(t+t_{n}, s-t-t_{n}\right)-v\left(t^{\prime}+t_{n}, s-t^{\prime}-t_{n}\right), \xi_{k}(s)\right) d s\right| \\
& \leq C\left|t-t^{\prime}\right|+\left|\int_{\mathbb{R}}\left(v\left(t+t_{n}, s-t-t_{n}\right)-v\left(t+t_{n}, s-t^{\prime}-t_{n}\right), \xi_{k}(s)\right) d s\right| \\
& =C\left|t-t^{\prime}\right|+\left|\int_{\mathbb{R}}\left(v\left(t+t_{n}, s-t_{n}\right), \xi_{k}(s+t)-\xi_{k}\left(s+t^{\prime}\right)\right)\right| d s \\
& \quad \leq C\left|t-t^{\prime}\right|+3 G v \kappa_{0}\left\|\xi_{k}-\tau_{t-t^{\prime}} \xi_{k}\right\|_{L^{1}}
\end{aligned}
$$

which, provided $k$ is fixed, goes to 0 when $t-t^{\prime}$ goes to 0 , uniformly in $n$. It follows that for any fixed $\varepsilon$ there is a $\delta$ such that $\left|t-t^{\prime}\right|<\delta$ implies that $d\left(v_{n}(t, \cdot), v_{n}\left(t^{\prime}, \cdot\right)\right)<\varepsilon$ for all $n$. This means that the set of functions $t \mapsto v_{n}(t, \cdot)$ is an equicontinuous family of functions in $C\left([-T, T], L^{\infty}(\mathbb{R}, H)_{\text {weak }^{*}}\right)$ for any $T$. Note that $\left|v_{n}(t, s)\right| \leq\|v\|_{X} / \kappa_{0}$, therefore, $v_{n}(t, \cdot)$ belongs to the ball of radius $\|v\|_{X} / \kappa_{0}$ in $L^{\infty}(\mathbb{R}, H)$, which by the Banach-Alaoglu theorem is compact in $L^{\infty}(\mathbb{R}, H)_{\text {weak* }}$. Using the ArzelaAscoli Theorem 7.3 and the diagonal process, we obtain a function $\bar{v}$ in $C\left(\mathbb{R}, L^{\infty}(\mathbb{R}, H)_{\text {weak }^{*}}\right)$ such that for some subsequence of $\left\{v_{n_{j}}\right\}$, (8.3) holds for any $T$. Without loss of generality, we assume that (8.3) holds for the sequence $\left\{v_{n}\right\}$.

To deal with the sequence $R_{n}(t, \cdot)=-B\left(v_{n}(t, \cdot)-\bar{v}(t, \cdot)\right)$ note that it is a composition of an equicontinuous family $\left\{v_{n}(t, \cdot)-\bar{v}(t, \cdot)\right\}$ in $C\left(\mathbb{R}, L^{\infty}\left(\mathbb{R}, P_{N} H\right)_{\text {weak }^{*}}\right)$ and a map $L^{\infty}\left(\mathbb{R}, P_{N} H\right)_{\text {weak }^{*}} \rightarrow L^{\infty}(\mathbb{R}, H)_{\text {weak }^{*}}$ induced by $B$. Since $P_{N} H$ is finite-dimensional, $B$ is a continuous quadratic form on $P_{N} H$, and hence the induced map is continuous. It follows that the family 
$R_{n}(t, \cdot) \in C\left(\mathbb{R}, L^{\infty}(\mathbb{R}, H)_{\text {weak }^{*}}\right)$ is also equicontinuous. Now note that by (A23),

$$
\begin{aligned}
\left|B\left(v_{n}(t, s)-\bar{v}(t, s)\right)\right| & \leq c_{\mathrm{A}}\left|v_{n}(t, s)-\bar{v}(s)\right|^{1 / 2}\left|A v_{n}(t, s)-A \bar{v}(s)\right|^{1 / 2}\left\|v_{n}(t, s)-\bar{v}(s)\right\| \\
& \leq c_{\mathrm{A}}\left(\kappa_{0} N\right)^{2}\left|v_{n}(t, s)-\bar{v}(s)\right|^{2} \leq 2 c_{\mathrm{A}}\left(\kappa_{0} N\right)^{2}\|v\|_{X},
\end{aligned}
$$

thus $R_{n}(t, \cdot)$ is in the ball of radius $2 c_{\mathrm{A}}\left(\kappa_{0} N\right)^{2}\|v\|_{X}$ in $L^{\infty}(\mathbb{R}, H)$. Therefore, again by Theorem 7.3 and the diagonal procedure, there exists $\bar{R}(t, \cdot) \in C\left(\mathbb{R}, L^{\infty}(\mathbb{R}, H)_{\text {weak }^{*}}\right)$ and a subsequence such that (8.4) holds for any $T$. Once again, without loss of generality, we assume that (8.3) and (8.4) hold for the whole sequence.

Finally, $w_{n}$ is equicontinuous in $C\left(\mathbb{R}^{2}, V\right)$ by Proposition 7.2, and for each $t, s w_{n}(t, s)$ is precompact in $V$ by (7.3), thus, by Theorem 7.3, (8.5) holds for some subsequence $v_{n_{j}}$.

The main result in this section is the following.

Proposition 8.2: Suppose that $N$ and $G$ satisfy (4.36). Let $\bar{v}, \bar{w}$, and $\bar{R}$ be as in Lemma 8.1. If $\bar{v}(t, s)$ and $\bar{w}(t, s)$ are both independent of $t$, then $\bar{R}$ is also independent of $t$ and

$$
\bar{u}(s)=\bar{v}(s)+\bar{w}(s)
$$

satisfies the Navier-Stokes equation

$$
\left.\frac{d}{d s} \bar{u}(s)+v A \bar{u}(s)+B(\bar{u}(s), \bar{u}(s))\right)=f+\bar{R}(s), \quad s \in \mathbb{R} .
$$

The function $\bar{R}$ can be viewed as the analog of the Reynolds stress due to averaging the NavierStokes equations in the theory of turbulence. ${ }^{15,17}$ Thus, $\bar{u}(\cdot)$ is a solution to the Navier-Stokes Equation (2.1) with the original force $f$, if and only if $\bar{v}(t, s)$ and $\bar{w}(t, s)$ are independent of $t$, and $\bar{R}(s)=0$. We do not know if any of these conditions are superfluous.

Proof: Using a change of variables, we write

$$
\begin{aligned}
w_{n}(t, s) & =w\left(t+t_{n}, s-\left(t+t_{n}\right)\right)=W\left(\tau_{t_{n}} v\right)\left(s-\left(t+t_{n}\right)\right) \\
& =\int_{-\infty}^{s-\left(t-t_{n}\right)} e^{-\nu\left(s-t-t_{n}-\sigma\right) A}\left[h-Q_{N} B\left(v\left(t+t_{n}, \sigma\right)+w\left(t+t_{n}, \sigma\right)\right)\right] d \sigma \\
& =(v A)^{-1} h-\int_{-\infty}^{s} e^{-v(s-\sigma) A}\left[Q_{N} B\left(v_{n}(t, \sigma)+w_{n}(t, \sigma)\right)\right] d \sigma .
\end{aligned}
$$

Now write

$$
\begin{aligned}
& \bar{w}(s)-(v A)^{-1} h+\int_{-\infty}^{s} e^{-v(s-\sigma) A} Q_{N}[B(\bar{v}(\sigma)+\bar{w}(\sigma))-\bar{R}(t, \sigma)] d \sigma \\
& =\bar{w}(s)-w_{n}(t, s)- \\
& \int_{-\infty}^{s} e^{-\nu(s-\sigma) A} Q_{N}\left[B\left(v_{n}(t, \sigma)+w_{n}(t, \sigma)\right)-B(\bar{v}(\sigma)+\bar{w}(\sigma))-B\left(v_{n}(t, \sigma)-\bar{v}(\sigma)\right)\right] d \sigma \\
& -\int_{-\infty}^{s} e^{-\nu(s-\sigma) A} Q_{N}\left[B\left(v_{n}(t, \sigma)-\bar{v}(\sigma)\right)+\bar{R}(t, \sigma)\right] d \sigma .
\end{aligned}
$$

Notice that

$$
\begin{aligned}
& B\left(v_{n}+w_{n}\right)-B(\bar{v}+\bar{w})-B\left(v_{n}-\bar{v}\right)= \\
& B_{\mathrm{sym}}\left(v_{n}+\bar{w}, w_{n}-\bar{w}\right)+B\left(w_{n}-\bar{w}\right)+B_{\mathrm{sym}}\left(\bar{v}+\bar{w}, v_{n}-\bar{v}\right),
\end{aligned}
$$

where

$$
B_{\text {sym }}\left(u_{1}, u_{2}\right)=B\left(u_{1}, u_{2}\right)+B\left(u_{2}, u_{1}\right) .
$$


Using (A23), (7.3), and (A22) as well, we find that

$$
\begin{aligned}
\left|B\left(v_{n}+\bar{w}, w_{n}-\bar{w}\right)\right| & \leq c_{\mathrm{A}}\left|v_{n}+\bar{w}\right|^{1 / 2}\left|A\left(v_{n}+\bar{w}\right)\right|^{1 / 2}\left\|w_{n}-\bar{w}\right\| \\
& \leq \frac{c_{\mathrm{A}}}{\kappa_{0}}\left(\left|A v_{n}\right|+|A \bar{w}|\right)\left\|w_{n}-\bar{w}\right\| \\
& \leq c_{\mathrm{A}}\left(N\|v\|_{X}+\frac{\Gamma}{\kappa_{0}}\right)\left\|w_{n}-\bar{w}\right\| \\
\left|B\left(w_{n}-\bar{w}, v_{n}+\bar{w}\right)\right| \leq & c_{\mathrm{L}}\left|w_{n}-\bar{w}\right|^{1 / 2}\left\|w_{n}-\bar{w}\right\|^{1 / 2}\left\|v_{n}+\bar{w}\right\|^{1 / 2}\left|A\left(v_{n}+\bar{w}\right)\right|^{1 / 2} \\
\leq & \frac{c_{\mathrm{L}}}{\kappa_{0} N^{1 / 2}}\left(\left|A v_{n}\right|^{2}+|A \bar{w}|^{2}\right)^{1 / 2}\left\|w_{n}-\bar{w}\right\| \\
\leq & \frac{c_{\mathrm{L}}}{\kappa_{0} N^{1 / 2}}\left(\kappa_{0} N\left\|v_{n}\right\|_{X}+|A \bar{w}|\right)\left\|w_{n}-\bar{w}\right\| \\
\leq & \frac{c_{\mathrm{L}}}{\kappa_{0} N^{1 / 2}}\left(\kappa_{0} N\|v\|_{X}+\Gamma\right)\left\|w_{n}-\bar{w}\right\| \\
\left|B\left(w_{n}-\bar{w}\right)\right| & \leq c_{\mathrm{A}}\left|w_{n}-\bar{w}\right|^{1 / 2}\left|A\left(w_{n}-\bar{w}\right)\right|^{1 / 2}\left\|w_{n}-\bar{w}\right\| \\
\leq & \frac{c_{\mathrm{A}}}{\kappa_{0} N}\left|A\left(w_{n}-\bar{w}\right)\right|\left\|w_{n}-\bar{w}\right\| \\
\leq & \frac{2 c_{\mathrm{A}}}{\kappa_{0} N} \Gamma\left\|w_{n}-\bar{w}\right\|,
\end{aligned}
$$

so that for each $t$,

$$
\int_{-\infty}^{s} e^{-v(s-\sigma) A} Q_{N}\left[B_{\mathrm{sym}}\left(v_{n}+\bar{w}, w_{n}-\bar{w}\right)+B\left(w_{n}-\bar{w}\right)\right] d \sigma \rightarrow 0 \quad \text { as } n \rightarrow \infty,
$$

by the Lebesgue dominated convergence theorem. Indeed, the integrand is bounded by

$$
2 \tilde{\tilde{\Gamma}}\|w\|_{Y} e^{-v(s-\sigma) \kappa_{0}^{2}},
$$

where

$$
\tilde{\tilde{\Gamma}}=\left(c_{\mathrm{A}} N+c_{\mathrm{L}} N^{1 / 2}\right)\|v\|_{X}+\left(\frac{c_{\mathrm{A}}}{\kappa_{0}}+\frac{c_{\mathrm{L}}}{\kappa_{0} N^{1 / 2}}+\frac{2 c_{\mathrm{A}}}{\kappa_{0} N}\right) \Gamma
$$

and goes to 0 at each $\sigma$ since $\left\|w_{n}(t, \sigma)-\bar{w}(\sigma)\right\| \rightarrow 0$ when $n \rightarrow \infty$.

Let $k \in D(A)$. Note that the function $\sigma \mapsto e^{-v(s-\sigma) A} k$ if $\sigma \leq s$ and $\sigma \mapsto 0$ if $\sigma>s$ is in $L^{1}(\mathbb{R}, H)$. Thus by (8.4),

$$
\int_{-\infty}^{s} e^{-v(s-\sigma) A}\left(Q_{N}\left(B\left(v_{n}(t, \sigma)-\bar{v}(\sigma)\right)+\bar{R}(t, \sigma)\right), k\right) d \sigma \rightarrow 0 \quad \text { as } n \rightarrow \infty .
$$

We also have

$$
\begin{aligned}
\left(\int_{-\infty}^{s} e^{-v(s-\sigma) A} Q_{N} B\left(\bar{v}+\bar{w}, v_{n}-\bar{v}\right) d \sigma, k\right) \\
=\int_{-\infty}^{s} Q_{N}\left(B\left(\bar{v}+\bar{w}, e^{-v(s-\sigma) A} k\right), v_{n}-\bar{v}\right) d \sigma .
\end{aligned}
$$

But by (A23) we have that, as a function of $\sigma$,

$$
B\left(\bar{v}(\sigma)+\bar{w}(\sigma), e^{-v(s-\sigma) A} k\right) \in L^{1}((-\infty, s], H) .
$$

We conclude that

$$
\int_{-\infty}^{s}\left(e^{-v(s-\sigma) A} Q_{N} B\left(\bar{v}+\bar{w}, v_{n}-\bar{v}\right), k\right) d \sigma \rightarrow 0 \quad \text { as } n \rightarrow \infty .
$$


For the other half of $B_{\text {sym }}\left(\bar{v}+\bar{w}, v_{n}-\bar{v}\right)$, we write

$$
\left(e^{-v(s-\sigma) A} B\left(v_{n}-\bar{v}, \bar{v}+\bar{w}\right), k\right)=\left(v_{n}-\bar{v}, \theta(\sigma)\right),
$$

where (due to (A24)) we have

$$
|\theta(\sigma)| \leq\|\bar{v}(\sigma)+\bar{w}(\sigma)\| e^{-v(s-\sigma) \kappa_{0}}|k|^{1 / 2}|A k|^{1 / 2} \quad \forall \sigma \leq s .
$$

It follows that $\theta(\sigma) \in L^{\infty}((-\infty, s])$, and hence

$$
\int_{-\infty}^{s}\left(e^{-v(s-\sigma) A} Q_{N} B\left(v_{n}-\bar{v}, \bar{v}+\bar{w}\right), k\right) d \sigma \rightarrow 0 \quad \text { as } n \rightarrow \infty .
$$

In particular, for $k \in D(A),(8.8)$ now implies that

$$
\left(\bar{w}(s)-(\nu A)^{-1} h+\int_{-\infty}^{s} e^{-v(s-\sigma) A} Q_{N}(B(\bar{v}(\sigma)+\bar{w}(\sigma))-\bar{R}(t, \sigma)) d \sigma, k\right)=0 .
$$

Since $D(A)$ is dense in $H$, we deduce that for each $t$,

$$
\bar{w}(s)=(v A)^{-1} h-\int_{-\infty}^{s} e^{-v(s-\sigma) A} Q_{N}(B(\bar{v}(\sigma)+\bar{w}(\sigma))-\bar{R}(t, \sigma)) d \sigma .
$$

Note that (8.12) implies that $Q_{N} \bar{R}(t, s)$ does not depend on $t$. Indeed, by the vector-valued version of the Lebesgue differentiation theorem we obtain that for each $t Q_{N} \bar{R}(t, s)=r(s)$ for almost all $s$,

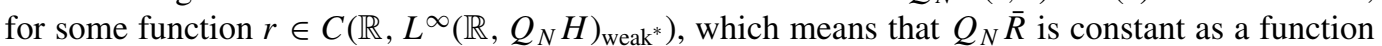
in $C\left(\mathbb{R}, L^{\infty}(\mathbb{R}, H)_{\text {weak }^{*}}\right)$.

Now observe that

$$
\begin{aligned}
v_{n}(t, s) & \left.=v\left(t+t_{n}, s-t-t_{n}\right)\right) \\
& =\int_{-\infty}^{t+t_{n}} e^{-v\left(t+t_{n}-\tau\right) A}\left[g-P_{N} B\left(v\left(\tau, s-t-t_{n}\right)+w\left(\tau, s-t-t_{n}\right)\right)\right] d \tau \\
& =\int_{-\infty}^{t} e^{-v(t-\tau) A}\left[g-P_{N} B\left(v\left(\tau+t_{n}, s-t-t_{n}\right)+w\left(\tau+t_{n}, s-t-t_{n}\right)\right)\right] d \tau \\
& =\int_{-\infty}^{t} e^{-v(t-\tau) A}\left[g-P_{N} B\left(v_{n}(\tau, s+\tau-t)+w_{n}(\tau, s+\tau-t)\right)\right] d \tau
\end{aligned}
$$

for all $t, s \in \mathbb{R}$; consequently

$$
\begin{aligned}
& \int_{S_{1}}^{S_{2}}\left(v_{n}(t, s), k\right) d s=\left((v A)^{-1} g, k\right)\left(S_{2}-S_{1}\right) \\
& \quad-\int_{-\infty}^{t}\left[\int_{S_{1}}^{S_{2}}\left(B\left(v_{n}(\tau, s+\tau-t)+w_{n}(\tau, s+\tau-t)\right), e^{-v(t-\tau) A} P_{N} k\right) d s\right] d \tau \\
& =\left((v A)^{-1} g, k\right)\left(S_{2}-S_{1}\right) \\
& \quad-\int_{-\infty}^{t}\left[\int_{S_{1}+\tau-t}^{S_{2}+\tau-t}\left(B\left(v_{n}(\tau, s)+w_{n}(\tau, s)\right), e^{-v(t-\tau) A} P_{N} k\right) d s\right] d \tau,
\end{aligned}
$$

where $k \in D(A), S_{1}<S_{2}$ are fixed (but otherwise arbitrary). By the convergence in (8.4), we can now pass to the limit in (8.13) by going through steps analogous to those from (8.7) to (8.12). Indeed, if 
$k$ is an eigenvector of $A$ with $A k=\lambda k$, then by (8.9),

$$
\begin{aligned}
& \int_{-\infty}^{t}\left[\int_{S_{1}+\tau-t}^{S_{2}+\tau-t}\left(B\left(v_{n}(\tau, s)+w_{n}(\tau, s)\right), e^{-v(t-\tau) A} P_{N} k\right) d s\right] d \tau \\
& =\int_{-\infty}^{t}\left[\int_{S_{1}+\tau-t}^{S_{2}+\tau-t}\left(B(\bar{v}+\bar{w})-\bar{R}, e^{-v(t-\tau) A} P_{N} k\right) d s\right] d \tau \\
& +\int_{-\infty}^{t}\left[\int_{S_{1}+\tau-t}^{S_{2}+\tau-t}\left(B_{\mathrm{sym}}\left(v_{n}+\bar{w}, w_{n}-\bar{w}\right)+B\left(w_{n}-\bar{w}\right), e^{-v(t-\tau) A} P_{N} k\right) d s\right] d \tau \\
& +\int_{-\infty}^{t} e^{-v(t-\tau) \lambda}\left[\int_{S_{1}+\tau-t}^{S_{2}+\tau-t}\left(B_{\mathrm{sym}}\left(\bar{v}+\bar{w}, v_{n}-\bar{v}\right), P_{N} k\right) d s\right] d \tau \\
& +\int_{-\infty}^{t} e^{-v(t-\tau) \lambda}\left[\int_{S_{1}+\tau-t}^{S_{2}+\tau-t}\left(\bar{R}+B\left(v_{n}-\bar{v}\right), P_{N} k\right) d s\right] d \tau .
\end{aligned}
$$

On the right-hand side the second, third, and fourth terms go to 0 as $n \rightarrow \infty$. In the second term, we use a bound similar to (8.10) and the Lebesgue dominated convergence theorem. For the third and fourth note, first that the integrands are bounded by $L^{1}$ functions, thus it suffices to show that for fixed $\tau$ the integrals

$$
\int_{S_{1}+\tau-t}^{S_{2}+\tau-t}\left(B_{\mathrm{sym}}\left(\bar{v}+\bar{w}, v_{n}-\bar{v}\right), k\right) d s
$$

and

$$
\int_{S_{1}+\tau-t}^{S_{2}+\tau-t}\left(\bar{R}+B\left(v_{n}-\bar{v}\right), k\right) d s
$$

go to 0 as $n \rightarrow \infty$. For the latter, note that the function $\chi_{\left[S_{2}+\tau-t, S_{1}+\tau-t\right](s)} k$ is in $L^{1}(\mathbb{R}, H)$, where $\chi_{I}$ is the characteristic function of an interval $I$, and use (8.4). For the former, write $\left(B_{\text {sym }}\left(\bar{v}+\bar{w}, v_{n}\right.\right.$ $-\bar{v}), k)=\left(v_{n}(t, s)-\bar{v}(s), \psi(s)\right)$ and note that, as above, by (A23) and (A24) $\chi_{\left[S_{2}+\tau-t, S_{1}+\tau-t\right]}$ $(s) \psi(s)$ is in $L^{1}(\mathbb{R}, H)$ and, therefore, we obtain the convergence by (8.3).

Setting $\bar{u}=\bar{v}+\bar{w}$, we obtain

$$
\begin{aligned}
& \int_{S_{1}}^{S_{2}}(\bar{v}(s), k) d s-\left((v A)^{-1} g, k\right)\left(S_{2}-S_{1}\right) \\
& =-\int_{-\infty}^{t}\left[\int_{S_{1}+\tau-t}^{S_{2}+\tau-t}\left(B(\bar{u}(s))-\bar{R}(\tau, s), e^{-v(t-\tau) A} P_{N} k\right) d s\right] d \tau \\
& =-\int_{-\infty}^{t}\left[\int_{S_{1}}^{S_{2}}\left(B(\bar{u}(s+\tau-t))-\bar{R}(\tau, s+\tau-t), e^{-v(t-\tau) A} P_{N} k\right) d s\right] d \tau \\
& =-\int_{S_{1}}^{S_{2}}\left(\int_{-\infty}^{t} e^{-v(t-\tau) A} P_{N}[B(\bar{u}(s+\tau-t))-\bar{R}(\tau, s+\tau-t)] d \tau, k\right) d s \\
& =-\left(\int_{S_{1}}^{S_{2}}\left\{\int_{-\infty}^{s} e^{-v(s-\sigma) A} P_{N}[B(\bar{u}(\sigma))-\bar{R}(t-s+\sigma, \sigma)] d \sigma\right\} d s, k\right) .
\end{aligned}
$$

Since for fixed $S_{1}<S_{2}, t$ in $\mathbb{R}, k$ can run over all linear combinations of eigenvectors of $A$, of which are dense in $H$, we deduce

$$
\int_{S_{1}}^{S_{2}}\left\{\bar{v}(s)-\int_{-\infty}^{s} e^{-v(s-\sigma) A}\left[g-P_{N} B(\bar{u}(\sigma))+P_{N} \bar{R}(t-s+\sigma, \sigma)\right] d \sigma\right\} d s=0
$$

for all $S_{1}<S_{2}, t$ in $\mathbb{R}$. 
The final function in braces above is in $L^{1}([-S, S], H)$ for all $S>0$. Therefore, the vector-valued version of Lebesgue's differentiation theorem now implies

$$
\bar{v}(s)=\int_{-\infty}^{s} e^{-v(s-\sigma) A}\left[g-P_{N} B(\bar{u}(\sigma))+P_{N} \bar{R}(t-s+\sigma, \sigma) d t\right] d \sigma
$$

for $s \notin \Sigma_{t}$, where the measure of $\Sigma_{t}$ is 0 . Note that since $C\left(\mathbb{R}, L^{\infty}(\mathbb{R}, H)\right)_{\text {weak }^{*}} \subset L_{\text {loc }}^{1}\left(\mathbb{R}^{2}\right)$, both the right- and left-hand sides of (8.14) are in $L_{\text {loc }}^{1}\left(\mathbb{R}^{2}\right)$, and therefore by Fubini's theorem (8.14) holds for almost all pairs $(t, s) \in \mathbb{R}^{2}$. By substituting $(t, s)$ with $(t+\tau, s+\tau)$ we obtain that for almost all $t, s$,

$$
\bar{v}(s+\tau)=\int_{-\infty}^{s+\tau} e^{-v(s+\tau-\sigma) A}\left[g-P_{N} B(\bar{u}(\sigma))+P_{N} \bar{R}(t-s+\sigma, \sigma) d t\right] d \sigma
$$

holds for almost all $\tau$. Fix such $t, s$ that (8.15) holds for almost all $\tau$. Then the right-hand side of (8.15) is continuous in $\tau$ and hence $\bar{v}$ coincides almost everywhere with continuous function. Thus, we can suppose that $\bar{v}$ is (absolutely) continuous. Taking the derivative with respect to $\tau$ of (8.15), we obtain that for almost all $(t, s) \in \mathbb{R}^{2}$,

$$
\frac{d \bar{v}(s+\tau)}{d s}+v A \bar{v}(s+\tau)+P_{N} B(\bar{u}(s+\tau), \bar{u}(s+\tau))=g+P_{N} \bar{R}(t+\tau, s+\tau)
$$

for almost all $\tau$. It follows that

$$
\frac{d \bar{v}(s)}{d s}+v A \bar{v}(s)+P_{N} B(\bar{u}(s), \bar{u}(s))=g+P_{N} \bar{R}(t, s)
$$

for almost all $t$, s. We, therefore, obtain that the function $\bar{R} \in C\left(\mathbb{R}, L^{\infty}(\mathbb{R}, H)_{\text {weak }^{*}}\right)$ is almost everywhere equal to some fixed element of $L^{\infty}(\mathbb{R}, H)$. Since $\bar{R}$ is continuous, this equality holds everywhere, thus $\bar{R}$ does not depend on $t$.

\section{STATIONARY SOLUTIONS OF (2.17)}

A solution $v(t, \cdot)$ is a stationary solution of (2.17) if and only if $v(t, s)=v_{0}(s)$ for all $t$ and

$$
\nu A v_{0}(s)=g-P_{N} B\left(\varphi v_{0}(s)+W\left(v_{0}\right)(s)\right),
$$

that is, if and only if it satisfies the following differential algebraic equation:

$$
\begin{gathered}
\nu A v_{0}(s)=g-P_{N} B\left(\varphi v_{0}(s)+w_{0}(s)\right), \\
\frac{d w_{0}(s)}{d s}+v A w_{0}(s)=h-Q_{N} B\left(\varphi v_{0}(s)+w_{0}(s)\right),
\end{gathered}
$$

where $w_{0}=W\left(v_{0}\right)$. In this case, the energy and enstrophy balances are

$$
\begin{aligned}
\frac{1}{2} \frac{d}{d s}\left|w_{0}\right|^{2}+v\left\|u_{0}\right\|^{2} & =\left(f, u_{0}\right), \\
\frac{1}{2} \frac{d}{d s}\left\|w_{0}\right\|^{2}+v\left|A u_{0}\right|^{2} & =\left(f, A u_{0}\right),
\end{aligned}
$$

where $u_{0}=v_{0}+w_{0}$ (compare to (A10) and (A11)).

It is shown in Ref. 10 that solutions on the global attractor of the NSE must satisfy $v\|u(t)\|^{2}$ $\leq|f||u(t)|$ for all $t \in \mathbb{R}$, with equality holding for some $t$ if and only if $f$ is an eigenvector of $A$. One might ask if at least the stationary solutions of (2.17) satisfy an analogous property. We will give below a partial answer for this case, which involves an application of (9.3) and (9.4). It is only a partial answer in that it holds only under the assumption that the $L^{2}$-norm of the high modes is nondecreasing. 
Let $u_{0}=v_{0}+w_{0}$. We first prove the following.

Lemma 9.1: Let $w_{0}$ and $v_{0}$ satisfy (9.1) and (9.2), and let $\sigma_{0}<\sigma_{1}$. Suppose that $\left|w_{0}(s)\right|$ is nondecreasing in $\left(\sigma_{0}, \sigma_{1}\right)$, then

(i) $\quad v\left\|u_{0}(s)\right\|^{2} \leq|f|\left|u_{0}(s)\right|$ for all $s \in\left(\sigma_{0}, \sigma_{1}\right)$.

(ii) If, in addition, $w_{0}(s)$ is nonzero for all $s \in\left(\sigma_{0}, \sigma_{1}\right),\left\|w_{0}(s)\right\|^{2} /\left|w_{0}(s)\right|$ is nondecreasing, and for some $s_{0} \in\left(\sigma_{0}, \sigma_{1}\right)$, we have $\left\|u_{0}\left(s_{0}\right)\right\|^{2} /\left|u_{0}\left(s_{0}\right)\right|=|f| / \nu$, then $f$ is an eigenvector of $A$ for the eigenvalue $\lambda^{0}=\left\|u_{0}\left(s_{0}\right)\right\|^{2} /\left|u_{0}\left(s_{0}\right)\right|^{2}, \lambda^{0}>\left(N \kappa_{0}\right)^{2}$, and $f=Q_{N} f$.

Proof: In the proof we drop the subscript on $w_{0}, v_{0}, u_{0}$, to simplify notation. Denote $\chi_{w}=\|w\|^{2} /|w|, \chi_{u}=\|u\|^{2} /|u|, \lambda_{w}=\|w\|^{2} /|w|^{2}, \lambda_{u}=\|u\|^{2} /|u|^{2}$. Since $w(s) \neq 0$ in $\left(\sigma_{0}, \sigma_{1}\right)$, these quantities are well-defined. From (9.3) and the fact that $|w|$ is nondecreasing it follows that $(f, u)-v\|u\|^{2} \geq 0$, which in turn implies that $\chi_{u}(s) \leq|f| / v$, proving (i).

Passing to the proof of (ii), we start by observing that from (9.3) and (9.4) it follows that:

$$
\begin{aligned}
& \chi_{w}(s)-\chi_{w}\left(s_{0}\right)= \\
& \int_{s_{0}}^{s} \frac{2 v}{|w|}\left[\left|\frac{f}{2 v}\right|^{2}-\frac{\chi_{u}^{2}}{4}-\left|\frac{f}{2 v}-\left(A-\frac{\lambda_{u}}{2}\right) u\right|^{2}-\frac{\lambda_{w}-\lambda_{u}}{2}\left(\left(\frac{f}{v}, u\right)-\|u\|^{2}\right)\right] d \sigma .
\end{aligned}
$$

By (i), since $|w|$ is nondecreasing, and the assumption $\chi_{u}\left(s_{0}\right)=|f| / \nu$, we deduce from (9.3) that $(f, u)=|f||u|$; hence we have $u\left(s_{0}\right)=c f$, where $c=|f|^{2} / v\|f\|^{2}$.

Since $\chi_{w}$ is assumed to be nondecreasing, the integrand in (9.5) is non-negative for almost all $\sigma$; also every summand in it is continuous, except for $\left|f / 2 v-\left(A-\lambda_{u} / 2\right) u\right|$, which is lower semicontinuous. This suffices, however, to ensure that the integrand is in fact non-negative at every point of $\left(\sigma_{0}, \sigma_{1}\right)$.

In particular, at the point $s_{0}$ we have that $\|u\|^{2}=(f / v, u)$, thus it follows that:

$$
\left|\frac{f}{2 v}-\left(A-\frac{\lambda_{u\left(s_{0}\right)}}{2}\right) u\left(s_{0}\right)\right|=0,
$$

hence $\left(A-\lambda^{0}\right) f=0$, where $\lambda^{0}=\lambda_{u\left(s_{0}\right)}$. Indeed,

$$
\left(A-\lambda^{0}\right) f=\left(A-\frac{\lambda^{0}}{2}\right) f-\frac{\lambda^{0}}{2} f=\frac{v\|f\|^{2}}{|f|^{2}}\left(A-\frac{\lambda^{0}}{2}\right) u\left(s_{0}\right)-\frac{\lambda^{0}}{2} f=\frac{\nu\|f\|^{2}}{|f|^{2}} \frac{f}{2 v}-\frac{\lambda^{0}}{2} f=0 .
$$

If $\lambda^{0} \leq\left(N \kappa_{0}\right)^{2}$ it would follow that $f=g$, which gives that $w\left(s_{0}\right)=0$, but this is contrary to our assumption. Thus, we have that $\lambda^{0}>\left(N \kappa_{0}\right)^{2}$, and so $f=h, g=0, v\left(s_{0}\right)=0, w\left(s_{0}\right)=h / v \lambda^{0}$, and $A h=\lambda^{0} h$.

Let $h_{0}=h / \nu \lambda^{0}$. Now we need the following lemma.

Lemma 9.2: Assume that $v_{0}$ and $w_{0}$ satisfy (9.1) and (9.2), and that $g=0$ and $h$ is an eigenvector of $A$. Then for large enough $N, v(s)=0$ and $w(s)=h_{0}$ for all $s$, where $\lambda^{0}$ is an eigenvalue of $A$.

Proof: Once again, denote $w_{0}, v_{0}, u_{0}$ by $w, v, u$ for simplicity. From (9.1), (A20), and (4.22), we obtain that

$$
\begin{aligned}
v\|v\|^{2} & =-(B(u, w), v)=-(B(w, w), v)+(B(v, v), w) \\
& \leq(B(w, w), v)+c_{T}\|v\|^{2}\|w\| \frac{(\ln e N)^{1 / 2}}{\kappa_{0} N},
\end{aligned}
$$

whence

$$
v\|v\|^{2}\left[1-c_{T} \frac{G \beta(G, N)}{N}(\ln e N)^{1 / 2}\right] \leq-(B(w, w), v) .
$$


By denoting $w=h_{0}+\delta$, we have

$$
\begin{aligned}
-(B(w, w), v) & =-(B(\delta, w), v)-\left(B\left(h_{0}, \delta\right), v\right) \\
& \leq(\ln e N)^{1 / 2}\|\delta\|\left(c_{T}|w|\|v\|+c_{B}\left|h_{0}\right|\|v\|\right) \\
& \leq(\ln e N)^{1 / 2}\left(c_{T} \frac{v G \beta(G, N)}{N}+c_{B} v \frac{\kappa_{0}^{2} G}{\lambda^{0}}\right)\|v\|\|\delta\| .
\end{aligned}
$$

From (9.7) and (9.8), we obtain

$$
\|v\|\left[1-c_{T} \frac{G \beta(G, N)}{N}(\ln e N)^{1 / 2}\right] \leq(\ln e N)^{1 / 2} \frac{G}{N}\left(c_{T} \beta(G, N)+c_{B} \frac{\kappa_{0}^{2} N}{\lambda^{0}}\right)\|\delta\| .
$$

Therefore, if

$$
N>2 c_{T} G \beta(G, N)(\ln e N)^{1 / 2},
$$

we have the bound

$$
\|v\| \leq c_{1}\|\delta\|
$$

where

$$
c_{1}=2(\ln e N)^{1 / 2} \frac{G}{N}\left(c_{T} \beta(G, N)+c_{B} \frac{1}{N}\right) .
$$

Now using (9.2) and (9.11), we obtain that

$$
\begin{aligned}
& \frac{1}{2} \frac{d}{d s}|\delta|^{2}+v\|\delta\|^{2}=-(B(u, u), \delta)=-\left(B\left(v+h_{0}+\delta, v+h_{0}\right), \delta\right) \\
& \quad=-\left(B\left(v+\delta, h_{0}\right), \delta\right)-(B(u, v), \delta) \\
& \quad \leq c_{T}(\ln e N)^{1 / 2}\|v\|\left\|h_{0}\right\||\delta|+c_{\mathrm{L}}|\delta|\|\delta\|\left\|h_{0}\right\|+c_{T}(\ln e N)^{1 / 2}\|u\|\|v\||\delta| \\
& \quad \leq v\left(c_{T} c_{1}(\ln e N)^{1 / 2}+c_{\mathrm{L}}\right) \frac{G}{N^{2}}\|\delta\|^{2}+c_{T} c_{1}(\ln e N)^{1 / 2} \frac{\|v\|_{X}+v \kappa_{0} G \beta(G, N)}{\kappa_{0} N}\|\delta\|^{2} .
\end{aligned}
$$

It follows from Lemma 3.1 and Theorem 2.3, that if $N$ is such that (9.10) holds and, moreover,

$$
\begin{aligned}
& N^{2}>2 G\left(c_{T} c_{1}(\ln e N)^{1 / 2}+c_{\mathrm{L}}\right), \\
& N>2 c_{T} c_{1}\left(G+c_{T}(\ln e N)^{1 / 2} G^{2}(6+\beta(G, N)) \beta(G, N)+G \beta(G, N)\right),
\end{aligned}
$$

then $\delta(s)=0$ for all $s$. Therefore from (9.11), we have for all $s$ that $v(s)=0$, and hence $u(s)$ $=h / \nu \lambda^{0}=w(s)$. Since $h$ is an eigenvector of $A$, it follows from (9.3) that $A h=\lambda^{0} h$.

Therefore, we obtain the following proposition.

Proposition 9.3: Suppose N satisfies (9.10) and (9.12). If the assumptions of Lemma 9.1 hold for $w_{0}=W\left(v_{0}\right)$ for some stationary solution $v_{0}$ of (2.17), then any stationary solution of (2.17) is zero.

We finish by simplifying the conditions on $N$ in Proposition 9.3. Suppose that $G \gg 1$ and

$$
N>C G(\ln e N)^{1 / 2}
$$

for some sufficiently large constant $C$. Then (9.10) holds and, moreover, $\beta(G, N) \leq 36 c_{T} / C$, and so $\beta(G, N)$ is small provided that $C$ is large enough. Choose $C$ such that $\beta(G, N)<1$. Then $c_{1}$ $\leq 2\left(c_{T}+c_{B}\right) / C$. Choose $C$, so that also $c_{1}<1$. Moreover, the bound in Theorem 2.3 is less than $\nu \kappa_{0}\left(G+2 c_{T} G^{2}(\ln e N)^{1 / 2}\right) \leq C_{2} G^{2}(\ln e N)^{1 / 2}$ for some constant $C_{2}$, which does not depend on $C$, since $G>>1$. We obtain then that the first inequality in (9.12) holds, and to satisfy the second inequality in (9.12), it suffices to take

$$
N>\varepsilon G^{2}(\ln e N)^{1 / 2}
$$


for any fixed $\varepsilon$ (provided that $G$ is large). Note that if (9.14) holds, then (9.13) also holds. It follows that conclusion of Proposition 9.3 is true as long as inequality (9.14) holds for $N$.

\section{ACKNOWLEDGMENTS}

This paper is dedicated to Professor Peter Constantin, on the occasion of his 60th birthday, as token of friendship and admiration for his contributions to research in partial differential equations and fluid mechanics.

The work of C.F. is supported in part by National Science Foundation (NSF) Grant No. DMS1109784, that of M.J. by DMS-1008661 and DMS-1109638, that of R. K. by the ERC starting Grant No. GA 257110 RaWG, and that of E.S.T. by DMS-1009950, DMS-1109640, and DMS-1109645, as well as the Minerva Stiftung/Foundation. The authors would like to thank the referee for several suggestions on how to rearrange the paper.

\section{APPENDIX: PRELIMINARIES ON NAVIER-STOKES EQUATIONS}

We gather in this section a number of well-known results for the 2D Navier-Stokes equations. The scalar product in the phase space $H$ is taken to be

$$
(u, v)=\int_{\Omega} u(x) \cdot v(x) d x, \quad \text { where } a \cdot b=a_{1} b_{1}+a_{2} b_{2}
$$

with associated $L^{2}$-norm

$$
|u|=(u, u)^{1 / 2}=\left(\int_{\Omega}|u(x)| d x\right)^{1 / 2},
$$

where, for a vector $a \in \mathbb{C}^{2}$, we denote its length by $|a|=\left(a \cdot a^{*}\right)^{1 / 2}$, with $a^{*}=\left(\overline{a_{1}}, \overline{a_{2}}\right)$. It will be clear from the context when $|\cdot|$ refers to the length of a vector in $\mathbb{C}^{2}$, as opposed to an $L^{2}$-norm.

The positive roots of the operator $A$ are defined by linearity from

$$
A^{\alpha} w_{j}=\lambda_{j}^{\alpha} w_{j}, \quad \text { for } j=0,1,2, \ldots
$$

on the domain

$$
D\left(A^{\alpha}\right)=\left\{u \in H: \sum_{j=0}^{\infty} \lambda_{j}^{2 \alpha}\left(u, w_{j}\right)^{2}<\infty\right\}
$$

for $\alpha \geq 0$.

Parseval's identity reads as

$$
|u|^{2}=L^{2} \sum_{k \in \mathbb{Z}^{2}} \hat{u}_{k} \cdot \hat{u}_{-k}=L^{2} \sum_{k \in \mathbb{Z}^{2}}\left|\hat{u}_{k}\right|^{2}
$$

as well as

$$
(u, v)=L^{2} \sum_{k \in \mathbb{Z}^{2}} \hat{u}_{k} \cdot \hat{v}_{-k}
$$

for $v=\sum \hat{v}_{k} e^{i \kappa_{0} k \cdot x}$.

We associate to each term in (2.5) a wave number $\kappa_{0}|k|$.

Recall the orthogonality relations of the bilinear term (see, e.g.,Refs. 6 and 30),

$$
\langle B(u, v), w\rangle=-\langle B(u, w), v\rangle, \quad u, v, w \in V,
$$

where $\langle\cdot, \cdot\rangle$ denotes the action of an element in $V^{\prime}$, as well as

$$
(B(u, u), A u)=0, \quad u \in D(A) .
$$

The strengthened form of the enstrophy invariance

$$
(B(A v, v), u)=(B(u, v), A v), \quad u \in V, \quad v \in D(A)
$$


is proved in Ref. 11. Relation (A4) together with (A2) implies

$$
\begin{aligned}
& (B(v, v), A u)+(B(v, u), A v)+(B(u, v), A v)=0, \quad u, v \in D(A), \\
& (B(A v, v), u)-(B(v, A v), u)+(B(v, v), A u)=0, \quad u, v \in D(A),
\end{aligned}
$$

see Ref. 15. We will also use Agmon's inequality

$$
\|u\|_{\infty} \leq c_{\mathrm{A}}|u|^{1 / 2}|A u|^{1 / 2}, \quad u \in D(A),
$$

and one of its consequences

$$
|(B(u, v), w)| \leq c_{\mathrm{A}}|u|\|v\|^{1 / 2}\left|A^{3 / 2} v\right|^{1 / 2}|w|, \quad u \in H, v \in D\left(A^{3 / 2}\right), w \in H .
$$

(See Refs. 6, 12, and 29).

In this paper, we will assume $f \in D\left(A^{j / 2}\right)$ for some specified $j \in\{0,1,2,3\}$, which is sufficient to guarantee that

$$
\frac{1}{2} \frac{d}{d t}|u|^{2}=\left(\frac{d u}{d t}, u\right), \quad \frac{1}{2} \frac{d}{d t}\|u\|^{2}=\left(\frac{d u}{d t}, A u\right)
$$

in the sense of distributions (see Lemma 1.2 in Chap. 3 in Ref. 29 and its proof). Taking the scalar product of (2.1) with $u$, respectively, $A u$, and applying (A2), (A3), and (A9) gives the energy, enstrophy balance equations

$$
\begin{aligned}
\frac{1}{2} \frac{d}{d t}|u|^{2}+v\|u\|^{2} & =(f, u), \\
\frac{1}{2} \frac{d}{d t}\|u\|^{2}+v|A u|^{2} & =(f, A u) .
\end{aligned}
$$

In the scientific literature,

$$
\kappa_{0}^{2}|u|^{2}=2 \text { times the total energy per unit mass }
$$

and

$$
\kappa_{0}^{2}\|u\|^{2}=\text { the total enstrophy per unit mass . }
$$

Straightforward applications of the Cauchy-Schwarz, Young, and Gronwall inequalities to (A11) gives

$$
\|u(t)\|^{2} \leq e^{-v \kappa_{0}^{2} t}\|u(0)\|^{2}+\frac{|f|^{2}}{v^{2} \kappa_{0}^{2}}\left(1-e^{-v \kappa_{0}^{2} t}\right) .
$$

If $|f|=0$, we have by (A12) that $\|u(t)\| \rightarrow 0$ as $t \rightarrow \infty$. Therefore, we assume throughout the paper that

$$
|f|>0 \text {. }
$$

It is clear from (A12) that the closed ball $\mathcal{B}$ of radius $2 \nu \kappa_{0} G$, centered at 0 , is absorbing and invariant, meaning that for any $u_{0}$ there exists $T=T\left(\left\|u_{0}\right\|\right)$ such that

$$
\left\|u\left(t ; u_{0}\right)\right\| \leq 2 v \kappa_{0} G, \quad \text { for all } t \geq T,
$$

and

$$
\left\|u\left(t ; u_{0}\right)\right\| \leq 2 \nu \kappa_{0} G, \quad \text { for all } t \geq 0, u_{0} \in \mathcal{B}
$$

It is well known (see, e.g., Ref. 15) that for the flow to be turbulent, we must have $G \gg 1$. While most of our results apply for all $G>0$, we will eventually assume $G \gg 1$. We also have from (A12) that

$$
\|u\| \leq \nu \kappa_{0} G \quad \text { for all } \quad u \in \mathcal{A}
$$


We often denote the solution as simply $u(t)$ when the choice of $u_{0}$ is irrelevant to our considerations. It is easily shown using (A12) that $T$, the time of absorption in (A14), can be taken to be

$$
T\left(\left\|u_{0}\right\|\right)=\frac{1}{v \kappa_{0}^{2}} \max \left\{1, \log \frac{\left\|u_{0}\right\|^{2}}{3 v^{2} \kappa_{0}^{2} G^{2}}\right\} .
$$

We will use the following bounds on the nonlinear term (see Refs. 10,17, and 31, and the references therein)

$$
\begin{gathered}
|(B(w, u), v)| \leq c_{T}\|w\|\|u\|\left(\ln \frac{e\|v\|}{\kappa_{0}|v|}\right)^{1 / 2}|v|, \\
|(B(w, u), v)| \leq c_{B}\|w\|\|u\|\left(\ln \frac{e|A w|}{\kappa_{0}\|w\|}\right)^{1 / 2}|v|, \\
|(B(w, u), v)| \leq c_{T}\|w\|\|u\|\left(\ln \frac{e|A u|}{\kappa_{0}\|u\|}\right)^{1 / 2}|v|, \\
|(B(u, v), w)| \leq c_{\mathrm{L}}|u|^{1 / 2}\|u\|^{1 / 2}\|v\||w|^{1 / 2}\|w\|^{1 / 2}, \\
|(B(u, v), w)| \leq c_{\mathrm{L}}|u|^{1 / 2}\|u\|^{1 / 2}\|v\|^{1 / 2}|A v|^{1 / 2}|w|, \\
|(B(u, v), w)| \leq c_{\mathrm{A}}|u|^{1 / 2}|A u|^{1 / 2}\|v\||w|, \\
|(B(u, v), w)| \leq c_{\mathrm{A}}|u|\|v\||w|^{1 / 2}|A w|^{1 / 2},
\end{gathered}
$$

which are valid whenever the norms involved make sense. One can use these inequalities to extend the domain of $B$ to larger functional spaces. For instance from (A23), one can infer that $B(u, v)$ can be extended by continuity to a map from $D(A) \times V$ to $H$. We will denote these extensions also by $B$.

\section{Proof of determining mode estimate}

We recall (see Chap. 3, Sec. 1 of Ref. 17) that $u=u_{1}-u_{2}$ satisfies

$$
\frac{1}{2} \frac{d}{d t}|u|^{2}+v\|u\|^{2}=-\left(B\left(u, u_{1}\right), u\right) \leq c_{\mathrm{L}}|u|\|u\|\left\|u_{1}\right\| \leq c_{\mathrm{L}}|u|\|u\| G v \kappa_{0},
$$

whence (since $P_{N} u=0$ and so $\kappa_{0} N|u| \leq\|u\|$ ),

$$
\begin{gathered}
\frac{d}{d t}|u|^{2}+2 v\|u\|^{2} \leq 2 c_{\mathrm{L}} \frac{G \nu \kappa_{0}}{\kappa_{0} N}\|u\|^{2}, \\
\frac{d}{d t}|u|^{2}+2 v N^{2} \lambda_{0}\left(1-\frac{G c_{\mathrm{L}}}{N}\right)|u|^{2} \leq 0,
\end{gathered}
$$

so that

$$
|u(t)|^{2} \leq\left|u\left(t_{0}\right)\right|^{2} e^{-2 v N^{2} \lambda_{0}\left(1-G c_{\mathrm{L}} / N\right)\left(t-t_{0}\right)}, \quad \forall t_{0} \leq t .
$$

Thus if

$$
N>c_{\mathrm{L}} G,
$$


we conclude (by letting $t_{0} \rightarrow-\infty$ ) that $u(t) \equiv 0$; that is if (A29) holds, then $P_{N}$ is determining. Note that (A29) implies that if $u_{1}(t)=u_{2}(t)$ only for all $t \leq T$, then still $u_{1}(t) \equiv u_{2}(t)$. In particular, $Q_{N} u(\cdot)$ is uniquely determined by $P_{N} u(\cdot)$ in case (A29) holds.

${ }^{1}$ Azouani, A., Olson, E., and Titi, E. S., "Continuous data assimilation using general interpolant observables," preprint.

${ }^{2}$ Azouani, A. and Titi, E. S., "Feedback control of nonlinear dissipative systems by finite determining parameters - A reaction-diffusion paradigm," preprint.

${ }^{3}$ Chueshov, I. D., "The theory of functionals that uniquely determine the asymptotic dynamics of infinite-dimensional dissipative systems," Usp. Mat. Nauk 53(4), 77-124 (1998).

${ }^{4}$ Cockburn, B., Jones, D. A., and Titi, E. S., "Estimating the number of asymptotic degrees of freedom for nonlinear dissipative systems," Math. Comput. 66(219), 1073-1087 (1997).

${ }^{5}$ Constantin, P. and Foias, C., "Global Lyapunov exponents, Kaplan-Yorke formulas and the dimension of the attractors for 2D Navier-Stokes equations," Commun. Pure Appl. Math. 38(1), 1-27 (1985).

${ }^{6}$ Constantin, P. and Foias, C., Navier-Stokes Equations, Chicago Lectures in Mathematics (University of Chicago, Chicago, IL, 1988).

${ }^{7}$ Constantin, P., Foias, C., Manley, O. P., and Temam, R., "Determining modes and fractal dimension of turbulent flows," J. Fluid Mech. 150, 427-440 (1985).

${ }^{8}$ Constantin, P., Foias, C., Nicolaenko, B., and Temam, R., "Integral manifolds and inertial manifolds for dissipative partial differential equations," Applied Mathematical Sciences Vol. 70 (Springer-Verlag, New York, 1989).

${ }^{9}$ Constantin, P., Foias, C., and Temam, R., "On the dimension of the attractors in two-dimensional turbulence," J. Phys. D 30(3), 284-296 (1988).

${ }^{10}$ Dascaliuc, R., Foias, C., and Jolly, M. S., "Relations between energy and enstrophy on the global attractor of the 2-D Navier-Stokes equations," J. Dyn. Differ. Equ. 17(4), 643-736 (2005).

${ }^{11}$ Dascaliuc, R., Foias, C., and Jolly, M. S., "Estimates on enstrophy, palinstrophy and invariant measures for 2-D tubulence," J. Differ. Equations 248, 792-819 (2010).

${ }^{12}$ Doering, C. R. and Gibbon, J. D., Applied Analysis of the Navier-Stokes Equations, Cambridge Texts in Applied Mathematics (Cambridge University Press, Cambridge, 1995).

${ }^{13}$ Foias, C., Jolly, M. S., and Kravchenko, R., "Determining forms for the Kuramoto-Sivashinsky and Lorenz equations: Analysis and computations," preprint.

${ }^{14}$ Foias, C., Jolly, M. S., Kravchenko, R., and Titi, E. S., "A unified approach to determining forms for the 2D Navier-Stokes equations - The general interpolants case," preprint.

${ }^{15}$ Foias, C., Jolly, M. S., Manley, O. P., and Rosa, R., "Statistical estimates for the Navier-Stokes equations and the Kraichnan theory of 2-D fully developed turbulence," J. Stat. Phys. 108(3-4), 591-645 (2002).

${ }^{16}$ Foias, C. and Kukavica, I., "Determining nodes for the Kuramoto-Sivashinsky equation,” J. Dyn. Differ. Equ. 7(2), 365-373 (1995).

${ }^{17}$ Foias, C., Manley, O., Rosa, R., and Temam, R., Navier-Stokes Equations and Turbulence, Encyclopedia of Mathematics and its Applications Vol. 83 (Cambridge University Press, Cambridge, 2001).

${ }^{18}$ Foias, C., Manley, O. P., Temam, R., and Trève, Y. M., "Asymptotic analysis of the Navier-Stokes equations," J. Phys. D 9(1-2), 157-188 (1983).

${ }^{19}$ Foias, C. and Prodi, G., "Sur le comportement global des solutions non-stationnaires des équations de Navier-Stokes en dimension 2," Rend. Semin. Matermatico Univ. di Padova 39, 1-34 (1967).

${ }^{20}$ Foias, C., Sell, G. R., and Temam, R., "Inertial manifolds for nonlinear evolutionary equations,” J. Differ. Equations 73(2), 309-353 (1988).

${ }^{21}$ Foias, C., Sell, G. R., and Titi, E. S., "Exponential tracking and approximation of inertial manifolds for dissipative nonlinear equations," J. Dyn. Differ. Equ. 1, 199-244 (1989).

${ }^{22}$ Foias, C. and Temam, R., "Asymptotic numerical analysis for the Navier-Stokes equations," in Nonlinear Dynamics and Turbulence, edited by J. Barenblatt (Pitman, Boston, 1983).

${ }^{23}$ Foias, C. and Temam, R., "Determination of the solutions of the Navier-Stokes equations by a set of nodal values," Math. Comput. 43, 117-133 (1984).

${ }^{24}$ Foias, C. and Titi, E. S., "Determining nodes, finite difference schemes and inertial manifolds," Nonlinearity 4, 135-153 (1991).

${ }^{25}$ Hale, J. K. and Raugel, G., "Regularity, determining modes and Galerkin methods," J. Math. Pures Appl. (9) 82(9), 1075-1136 (2003)

${ }^{26}$ Hayden, K., Olson, E., and Titi, E. S., "Discrete data assimilation in the Lorenz and 2D Navier-Stokes equations," J. Phys. D 240(18), 1416-1425 (2011).

${ }^{27}$ Jones, D. A. and Titi, E. S., "Upper bounds on the number of determining modes, nodes, and volume elements for the Navier-Stokes equations," Indiana Univ. Math. J. 42(3), 875-887 (1993).

${ }^{28}$ Olson, E. and Titi, E. S., "Determining modes for continuous data assimilation in 2D turbulence," J. Stat. Phys. 113(5-6), 799-840 (2003); Workshop on Progress in Statistical Hydrodynamics, Santa Fe, NM, 2002.

${ }^{29}$ Temam, R., Navier-Stokes Equations: Theory and Numerical Analysis, 3rd ed. (AMS Chelsea, 1984).

${ }^{30}$ Temam, R., "Infinite-dimensional dynamical systems in mechanics and physics," 2nd ed., Applied Mathematical Sciences Vol. 68 (Springer-Verlag, New York, 1997).

${ }^{31}$ Titi, E. S., "On a criterion for locating stable stationary solutions to the Navier-Stokes equations," Nonlinear Anal. 11(9), 1085-1102 (1987). 